\title{
GeTallele: a method for integrative analysis and visualization of DNA and RNA allele frequencies
}

\author{
Piotr Słowiński ${ }^{1, \#, *}$, Muzi Li ${ }^{2, \#, ~ P a u l a ~ R e s t r e p o, ~}{ }^{2,3}$, Nawaf Alomran², Liam F. Spurr ${ }^{2,4,5}$, \\ Christian Miller ${ }^{2}$, Krasimira Tsaneva-Atanasova ${ }^{1,6}$ and Anelia Horvath ${ }^{2,7,8}$
}

${ }^{1}$ Department of Mathematics, College of Engineering, Mathematics and Physical Sciences, Living Systems Institute, Translational Research Exchange @ Exeter and EPSRC Centre for Predictive Modelling in Healthcare, University of Exeter, EX4 4QJ Exeter, UK, ${ }^{2}$ McCormick Genomics and Proteomics Center, School of Medicine and Health Sciences, The George Washington University, 20037 Washington, DC, USA, ${ }^{3}$ Department of Genetics \& Genomics Sciences, Icahn School of Medicine at Mount Sinai, New York, NY 10029, ${ }^{4}$ Cancer Program, Broad Institute of MIT and Harvard, Cambridge, MA, ${ }^{5}$ Medical Oncology, Dana-Farber Cancer Institute, Boston, MA, ${ }^{6}$ Department of Bioinformatics and Mathematical Modelling, Institute of Biophysics and Biomedical Engineering, Bulgarian Academy of Sciences, 1113 Sofia, Bulgaria, ${ }^{7}$ Department of Pharmacology and Physiology, School of Medicine and Health Sciences, The George Washington University, 20037 Washington, DC, USA, ${ }^{8}$ Department of Biochemistry and Molecular Medicine, School of Medicine and Health Sciences, The George Washington University, 20037 Washington, DC, USA.

* To whom correspondence should be addressed.

\# Equal contribution

\begin{abstract}
Background: Asymmetric allele expression typically indicates functional and/or structural features associated with the underlying genetic variants. When integrated, RNA and DNA allele frequencies can reveal patterns characteristic of a wide-range of biological traits, including ploidy changes, genome admixture, allele-specific expression and gene-dosage transcriptional response.
\end{abstract}

Results: To assess RNA and DNA allele frequencies from matched sequencing datasets, we introduce a method for generating model distributions of variant allele frequencies (VAF) with a given variant read probability. In contrast to other methods, based on whole sequences or single SNV, proposed methodology uses continuous multi-SNV genomic regions. The methodology is implemented in a GeTallele toolbox that provides a suite of functions for integrative analysis, statistical assessment and visualization of Genome and Transcriptome allele frequencies. Using model VAF probabilities, GeTallele allows estimation and comparison of variant read probabilities (VAF distributions) in a sequencing dataset. We demonstrate this functionality across cancer DNA and RNA sequencing datasets.

Conclusion: Based on our evaluation, variant read probabilities can serve as a dependable indicator to assess gene and chromosomal allele asymmetries and to aid calls of genomic events in matched sequencing RNA and DNA datasets.

Contact: P.M.Slowinski@exeter.ac.uk

\section{Introduction}

RNA and DNA carry and present genetic variation in related yet distinct manners; the differences being informative of functional and structural traits. In diploid organisms, an important measure of genetic variation is the allele frequency, which can be measured from both genome (DNA) and transcriptome (RNA) sequencing data (encoded and expressed allele frequency, respectively). Differential DNA-RNA allele frequencies are associated with a variety of biological processes, such as genome admixture or allele-specific transcriptional regulation (Ferreira, et al., 2016; Ha, et al., 2012; Han, et al., 2015; Movassagh, et al., 2016; Shah, et al., 2012).

Most of the RNA-DNA allele comparisons from sequencing have been approached at nucleotide level, where it has proven to be highly informative for determining the alleles' functionality (Ferreira, et al., 2016; Ha, et al., 2012; Han, et al., 2015; Macaulay, et al., 2016; Morin, et al., 2013; Movassagh, et al., 2016; Reuter, et al., 2016; Shah, et al., 2012; Shi, et al., 2016; Shlien, et al., 2016; The, et al., 2012; Yang, et al., 2016). Comparatively, integration of allele signals at the molecular level, as derived from linear DNA and RNA carriers, is less explored due to challenges presented by short sequencing length. The different molecular nature of RNA and DNA also leads to limited compatibility of the sequencing output.

Herein, we address some of the above challenges by employing a mathematical model to assess differences between RNA- and DNA- variant allele frequencies (VAF) at single nucleotide variant (SNV) positions in the genome. To do that, we introduce GeTallele: a toolbox that provides a suit of functions for integrative analysis and visualization of Genome $\left(\mathrm{NAF}_{\mathrm{DNA}}\right)$ and Transcriptome $\left(\mathrm{VAF}_{\mathrm{RNA}}\right)$ allele frequencies along 
bioRxiv preprint doi: https://doi.org/10.1101/491209; this version posted April 23,2020 . The copyright holder for this preprint (which was not certified by peer review) is the author/funder, who has granted bioRxiv a license to display the preprint in perpetuity. It is made available under aCC-BY 4.0 International license.

genes and chromosomes. Using VAF, GeTallele infers possible copy number alterations (CNAs), and, at positions of interest, mathematically and statistically compares $\mathrm{VAF}_{\mathrm{RNA}}$ and $\mathrm{VAF}_{\mathrm{DNA}}$ distributions. Furthermore, GeTallele supports visualization of the allele distribution at a desired resolution - from nucleotide to genome. In contrast to other CNA-modelling methods based on statistical models, GeTallele is based on a mechanistic model of VAF distributions.

\section{Results}

We demonstrate GeTallele's functionality using sequencing DNA and RNA datasets from paired normal and tumour tissue obtained from 72 female patients with breast invasive carcinoma (BRCA) from The Cancer Genome Atlas (TCGA). Each dataset contains four matched sequencing sets: normal exome (Nex), normal transcriptome (Ntr), tumour exome (Tex), and tumour transcriptome (Ttr). The raw sequencing data were processed as previously described (Movassagh, et al., 2016) to generate the inputs for GetAllele.

The overall workflow of GetAllele is shown on Figure 1. As an input, GeTallele requires the absolute number of sequencing reads bearing the variant and reference nucleotide in each single-nucleotide variant (SNV) position across the four matched datasets. For each sample, to select SNV positions for analysis, we start with the high quality heterozygous SNV calls on the normal exome (Li, et al., 2009). In each of these positions we estimate the counts of the variant and reference reads ( $n_{\text {VAR }}$ and $n_{R E F}$, respectively) across the 4 matching datasets, and retain for further analyses only positions covered by a minimum total (variant and reference) required unique sequencing reads across. This threshold is flexible and is required to ensure that only sufficiently covered positions will be analysed; it is set to 3 in the herein presented results.

From each of the 4 matched datasets GetAllele estimates VAF based on $n_{\text {VAR }}$ and $n_{\text {REF }}$ covering the positions of interest: $V A F=n_{V A R} /\left(n_{V A R}+n_{R E F}\right)$. An example of genome-wide VAF values estimated from Tex, and their corresponding distribution of VAF values is shown in Figure 2. 
bioRxiv preprint doi: https://doi.org/10.1101/491209; this version posted April 23, 2020. The copyright holder for this preprint (which was not certified by peer review) is the author/funder, who has granted bioRxiv a license to display the preprint in perpetuity. It is made available under aCC-BY 4.0 International license.

A

INPUT DATA:
- $\mathrm{n}_{\text {ref }}$ reference counts
- $\mathrm{n}_{\text {var }}$ variant counts
- additional data

PROCESSING:
- compute VAFs
- generate model VAF
distributions
- divide data
into segments
- for each signal in each
segment estimate
variant probability $\left(\mathrm{V}_{\mathrm{PR}}\right)$

ANALYSIS:
- of $\mathrm{V}_{\mathrm{PR}}$ values in signals
- comparison of $\mathrm{V}_{\mathrm{PR}}$
values between
aligned signals
- comparison of $\mathrm{v}_{\mathrm{PR}}$
values with other data

VISUALISATION:
- of $\mathrm{V}_{\mathrm{PR}}$ values in a
segment
- Circos plots of whole
dataset
- Circos plots of a single
signal from the dataset

C

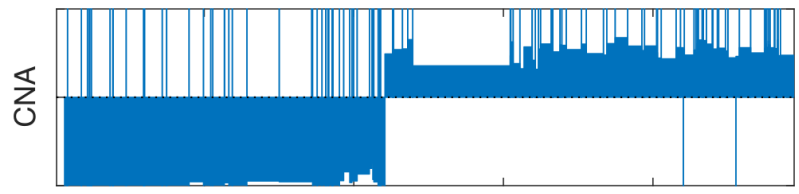

D

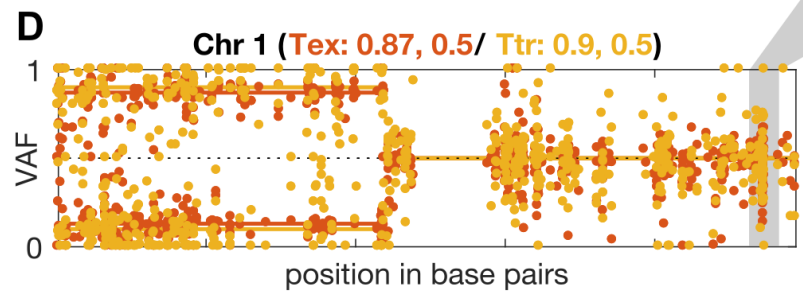

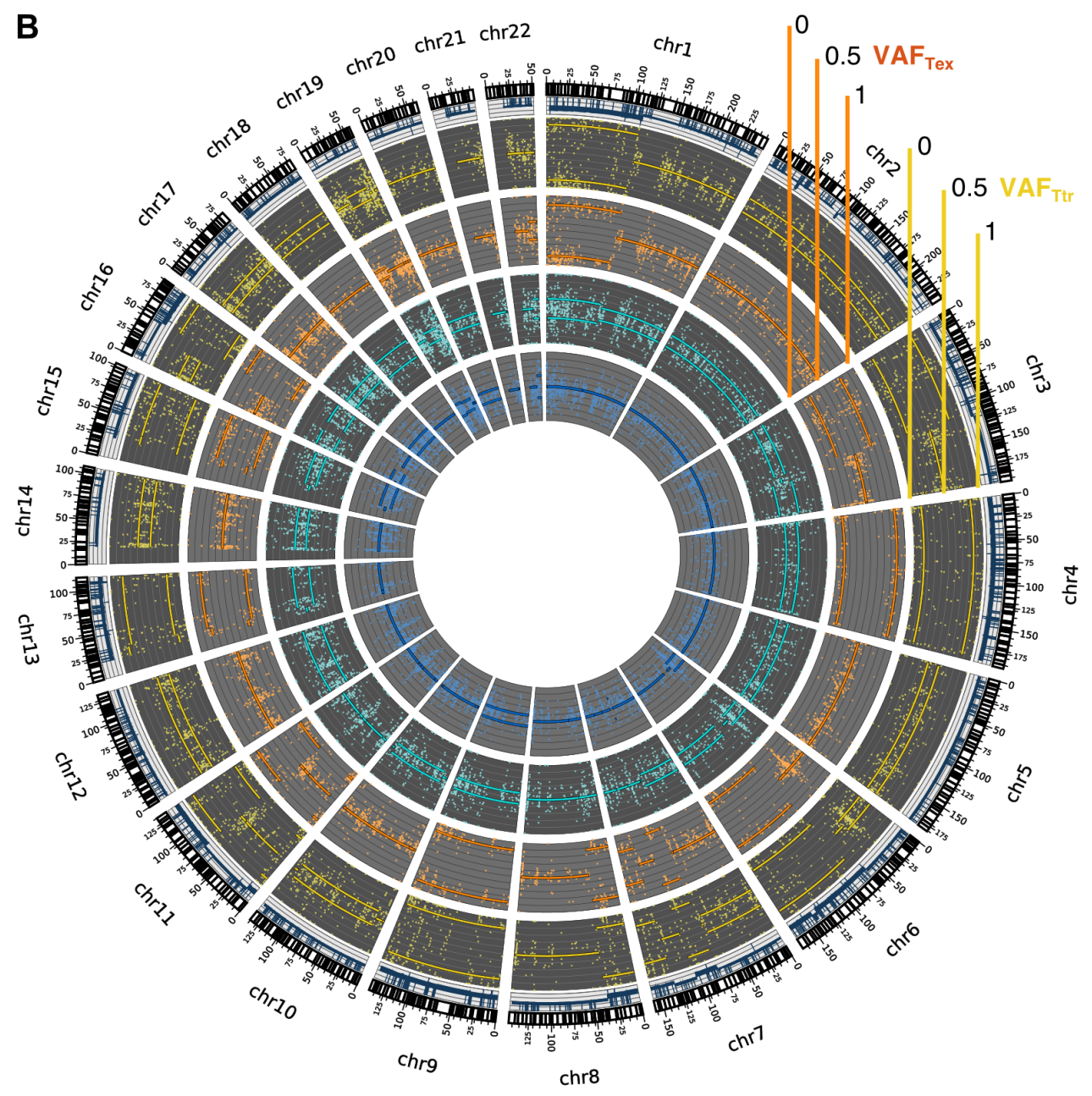

E

Fig. 1. GeTallele and visualisation of VAF data. A Toolbox description. B Visualisation of the whole dataset on the level of genome using Circos plot (blue - normal exome, cyan - normal transcriptome, orange - tumour exome, yellow - tumour transcriptome). C - $\mathrm{F}$ show in details VAFTEX and VAFTR values of chromosome 1; C - F Visualization of the VAF values with fitted variant probability (VPR - see Section 3.1 and Figure 2) values at the level of chromosome (D), custom genome region (E) and gene (F), for the chromosome level shown also are CNA values (C). Panel D shows that there are two segments with different VAF distributions. Panel $\mathrm{C}$ shows that change in the CNA is concurrent with the change in the VAF distributions. Tex - tumour exome (orange); Ttr - tumour transcriptome (yellow). 
bioRxiv preprint doi: https://doi.org/10.1101/491209; this version posted April 23, 2020. The copyright holder for this preprint (which was

A

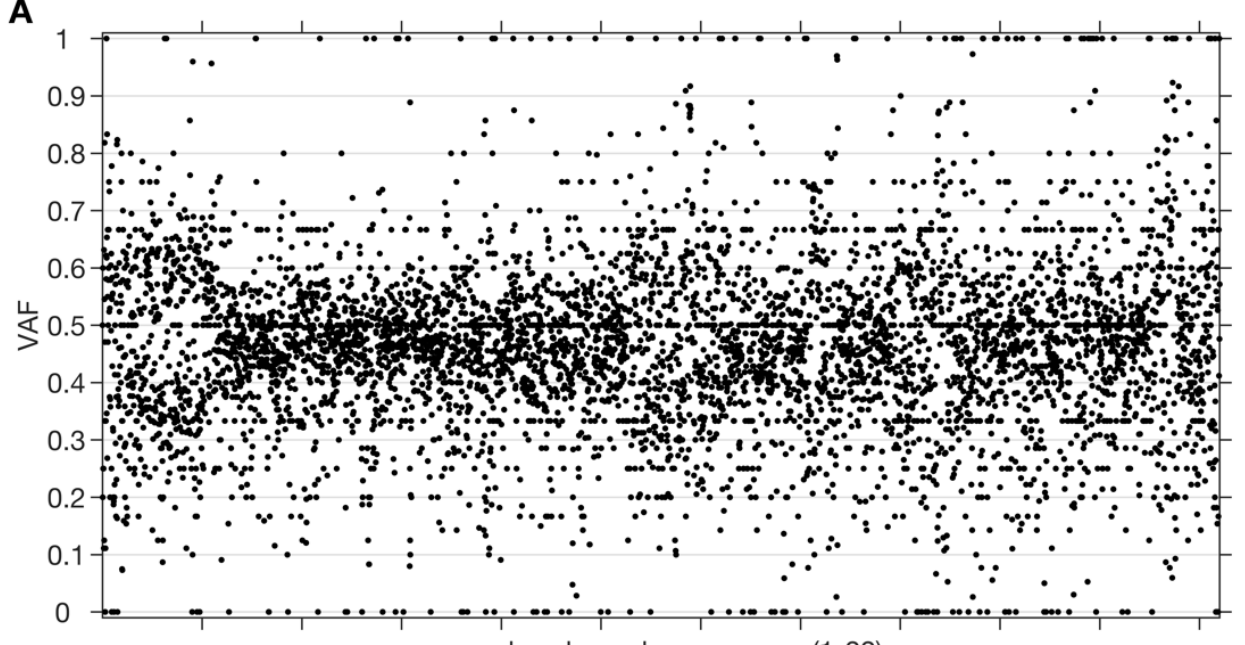

B

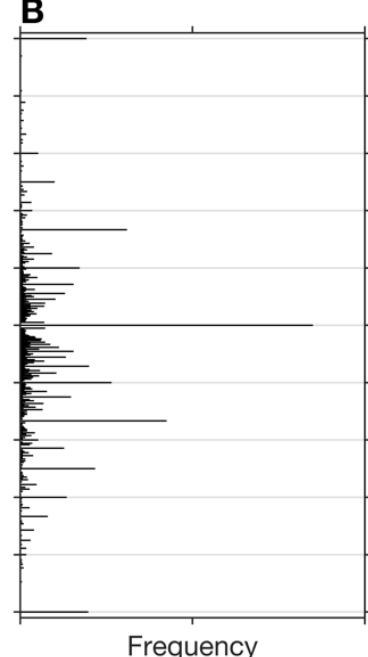

Fig. 2. Sample and distribution of variant allele frequencies (VAF) values. A. All the VAF values of a tumour exome sequencing signal (chromosomes 1-22) from one of the datasets. B. Histogram of the VAF values from panel A. Centres of bins of the histogram are located at elements of a Farey sequence.

Analysis of the $\mathrm{VAF}_{\mathrm{RNA}}$ and $\mathrm{VAF}_{\mathrm{DNA}}$ in the GeTallele is based on comparing probability of observing a given VAF value at various positions of interest. Estimation of the variant allele probability $\left(V_{P R}\right)$ is implemented using a data-driven mathematical model of distribution of the VAF values and is the core functionality of the GeTallele.

\subsection{Estimation of variant probability $\mathrm{v}_{\mathrm{pr}}$}

\subsubsection{Variant probability}

GetAllele aggregates frequency counts of a VAF sample into variant probability, $V_{\mathrm{PR}}$ - probability of observing a variant allele. The $V_{P R}$ is a single informative value indicative of copy number variation. The $V_{P R}$ is a parameter that describes the genomic event that through the sequencing process was transformed into a specific distribution of VAF values found in the signal. For example, in VAF $F_{D A}$ from a diploid genome, variant probability $\mathrm{V}_{\mathrm{PR}}=0.5$ (meaning that both alleles are equally probable) corresponds to a true allelic ratio of 1:1 for heterozygote sites. For heterozygote sites in the DNA from diploid monoclonal sample, the corresponding tumour $\mathrm{VAF}_{\mathrm{DNA}}$ is expected to have the following interpretations: $\mathrm{V}_{\mathrm{PR}}=1$ or $\mathrm{V}_{\mathrm{PR}}=0$ corresponds to a monoallelic status resulting from a deletion, and $\mathrm{VPR}_{\mathrm{PR}}=0.8$ (or 0.2), 0.75 (or 0.25), 0.67 (or 0.33) correspond to allelespecific tetra-, tri-, and duplication of the variant-bearing allele, respectively.

The $\mathrm{V}_{\mathrm{PR}}$ of the $\mathrm{VAF}_{\mathrm{RNA}}$ is interpreted as follows. In positions corresponding to DNA heterozygote sites, alleles not preferentially targeted by regulatory traits are expected to have expression rates with variant probability $V_{P R}=0.5$, which (by default) scale with the DNA allele distribution. Differences between $V_{A} F_{D N A}$ and $V_{A F} F_{R N A}$ values are observed in special cases of transcriptional regulation where one of the alleles is preferentially transcribed over the other. In the absence of allele-preferential transcription, $V \mathrm{FF}_{\mathrm{DNA}}$ and $\mathrm{VAF}_{\mathrm{RNA}}$ are anticipated to have similar $V_{P R}$ across both diploid (normal) and aneuploid (affected by CNAs) genomic regions. Consequently, $V F_{D N A}$ and $V_{A} F_{R N A}$ are expected to synchronously switch between allelic patterns along the chromosomes, with the switches indicating break points of DNA deletions or amplifications.

Since we observed that DNA and RNA signals have different distributions of total reads and also that the distributions of total reads vary between participants, the model VAF distributions are generated individually for each sequencing signal and each participant.

To estimate $V_{P R}$ in the signals, GeTallele first generates model VAF distributions and then uses the earth mover's distance (EMD) (Kantorovich and Rubinstein, 1958; Levina and Bickel, 2001) to fit them to the data. To generate a model VAF distribution with a given variant probability, VPR, GeTallele, bootstraps 10000 values of the total reads (sum of the variant and reference reads; $n_{V A R}+n_{R E F}$ ) from the analysed signal in the dataset. It then uses binomial pseudorandom number generator to get number of successes for given number of total reads and a given value of $V_{P R}$ (implemented in the Matlab function binornd). The $V_{P R}$ is the probability of success and generated number of successes is interpreted as an nVAR. Since the model $v_{P R}$ can take any value, it can correspond to a single genomic event as well as any combination of genomic events in any mixture of normal and tumour populations (See section 2.1.3). 
bioRxiv preprint doi: https://doi org/101101/491209; this version posted April 23, 2020. The copyright holder for this preprint (which was not certified by peer review) is the author/funder, who has granted bioRxiv a license to display the preprint in perpetuity. It is made available under aCC-BY 4.0 International license.

The analysis presented in the paper uses 51 model VAF distributions with $V_{\mathrm{PR}}$ values that vary from 0.5 to 1 with step (increment of) 0.01 . The model VAF distributions are parametrized using only $V_{P R} \geq 0.5$, however, to generate them we use $V_{P R}$ and its symmetric counterpart $1-V_{P R}$. The process of generating model VAF distributions and examples of model and real VAF distributions with different values of $\mathrm{VPR}_{\mathrm{PR}}$ are illustrated in Fig. 3.

A

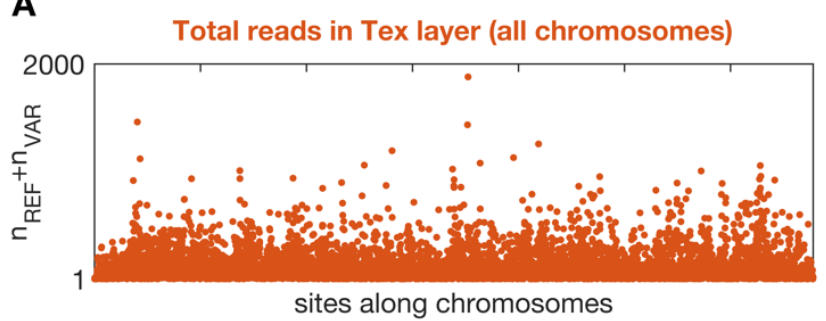

Distribution of total reads in the Tex layer

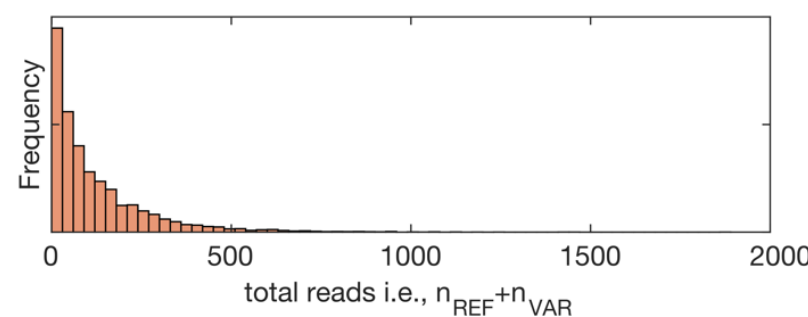

Generate N new total reads by resampling with replacement from one of the data layers.

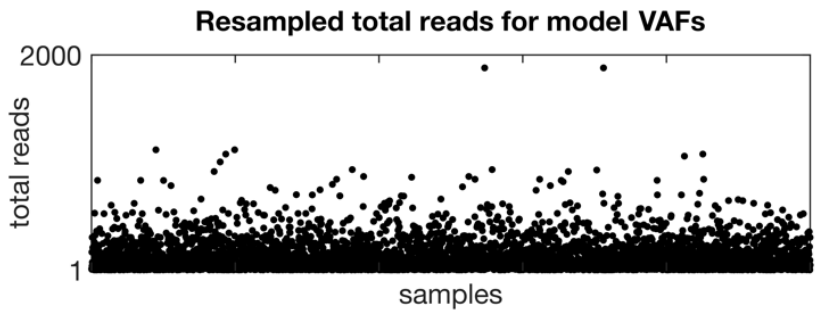

Use half of the new total reads to model $n_{\text {REF }}$ and the other half to model $n_{\text {VAR }}$.

Each total read value is used to model individual $n_{R E F, i}$ or $n_{V A R, i}$. They are modelled as random numbers from $\operatorname{Bin}\left(\right.$ total $\left._{i}, 1-v_{p r}\right)$ and $B i n\left(\right.$ total $\left._{i}, v_{p r}\right)$, respectively.

Convert the read counts into VAFs, $V A F_{i}=n_{i} /$ total $_{i}$, and combine the reference and variant values to get model VAF distributions.
B

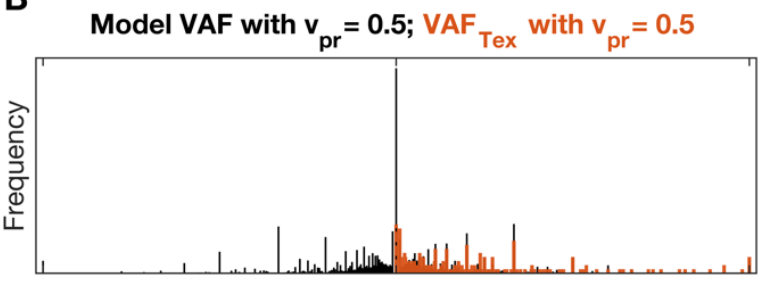

C

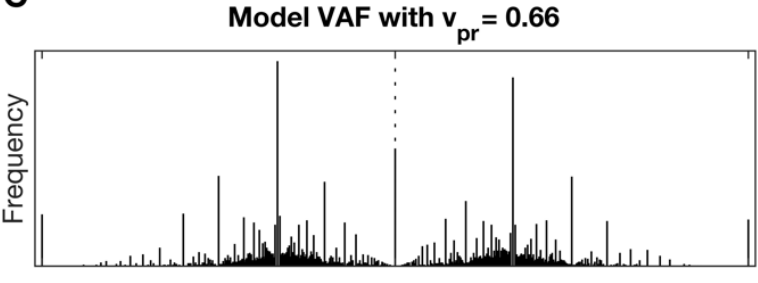

D

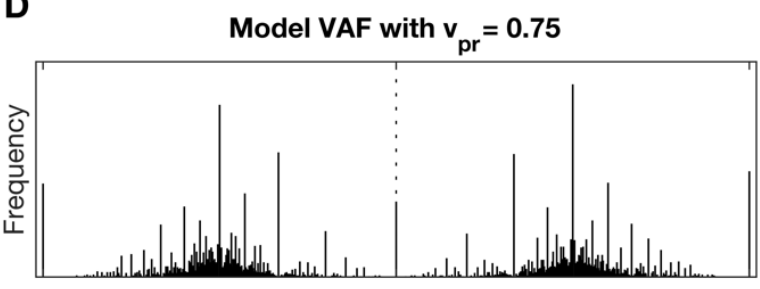

E

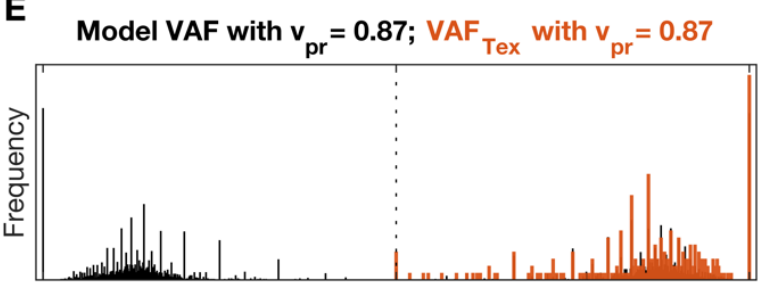

$\mathbf{F}$

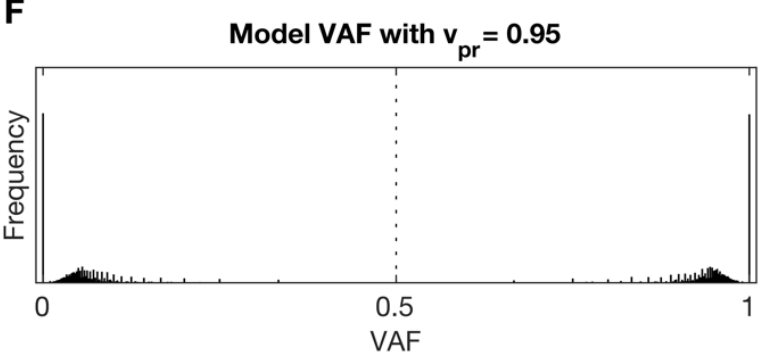

Fig. 3. Model and real VAF distributions. A Description of the model used to generate model VAF distributions, $\operatorname{Bin}(n, p)$ stands for binomial distribution with parameters $n$ (number of trials) and $p$ (probability of success). B - F Model VAF distributions for different values of VPR. Panels $B$ and $F$ show additionally distributions of VAFTEX for the two windows shown in Figure 1D.

\subsubsection{V $V_{\mathrm{PR}}$ estimation}

Earth mover's distance (EMD) is a metric for quantifying differences between probability distributions (Kantorovich and Rubinstein, 1958; Levina and Bickel, 2001) and in the case of univariate distributions it can be computed as:

$$
\operatorname{EMD}\left(\mathrm{PDF}_{1}, \mathrm{PDF}_{2}\right)=\int_{Z}\left|C D F_{1}(z)-C D F_{2}(z)\right| d z .
$$


bioRxiv preprint doi: https://doi.org/10.1101/491209; this version posted April 23, 2020. The copyright holder for this preprint (which was not certified by peer review) is the author/funder, who has granted bioRxiv a license to display the preprint in perpetuity. It is made available under aCC-BY 4.0 International license.

Here, $\mathrm{PDF}_{1}$ and $\mathrm{PDF}_{2}$ are two probability density functions and $\mathrm{CDF}_{1}$ and $\mathrm{CDF}_{2}$ are their respective cumulative distribution functions. $Z$ is the support of the PDFs (i.e. set of all the possible values of the random variables described by them). Because VAFs are defined as simple fractions with values between 0 and 1, their support is given by a Farey sequence (Hardy, et al., 2008) of order $n$; $n$ is the highest denominator in the sequence. For example, Farey sequence of order 2 is $0,1 / 2,1$ and Farey sequence of order 3 is $0,1 / 3,1 / 2,2 / 3,1$. GeTallele uses a Farey sequence of order 1000 as the support Z for all computations involving EMD.

To estimate $V_{P R}$, GeTallele computes EMD between the distribution of the VAF values of each signal in the window and the 51 model VAF distributions (i.e. observed vs modelled VAF), the estimate is given by the $V_{P R}$ of the model VAF distribution that is closest to the VAF distribution in the window. Examples of VAF distributions with fitted model VAF distributions are shown in Figure 3A and D. The dependence of the confidence intervals of the estimation on the number of VAF values in a window is illustrated in Fig. 4, which clearly demonstrates that the larger the number of VAFs in the chosen window the higher the accuracy of the estimate.
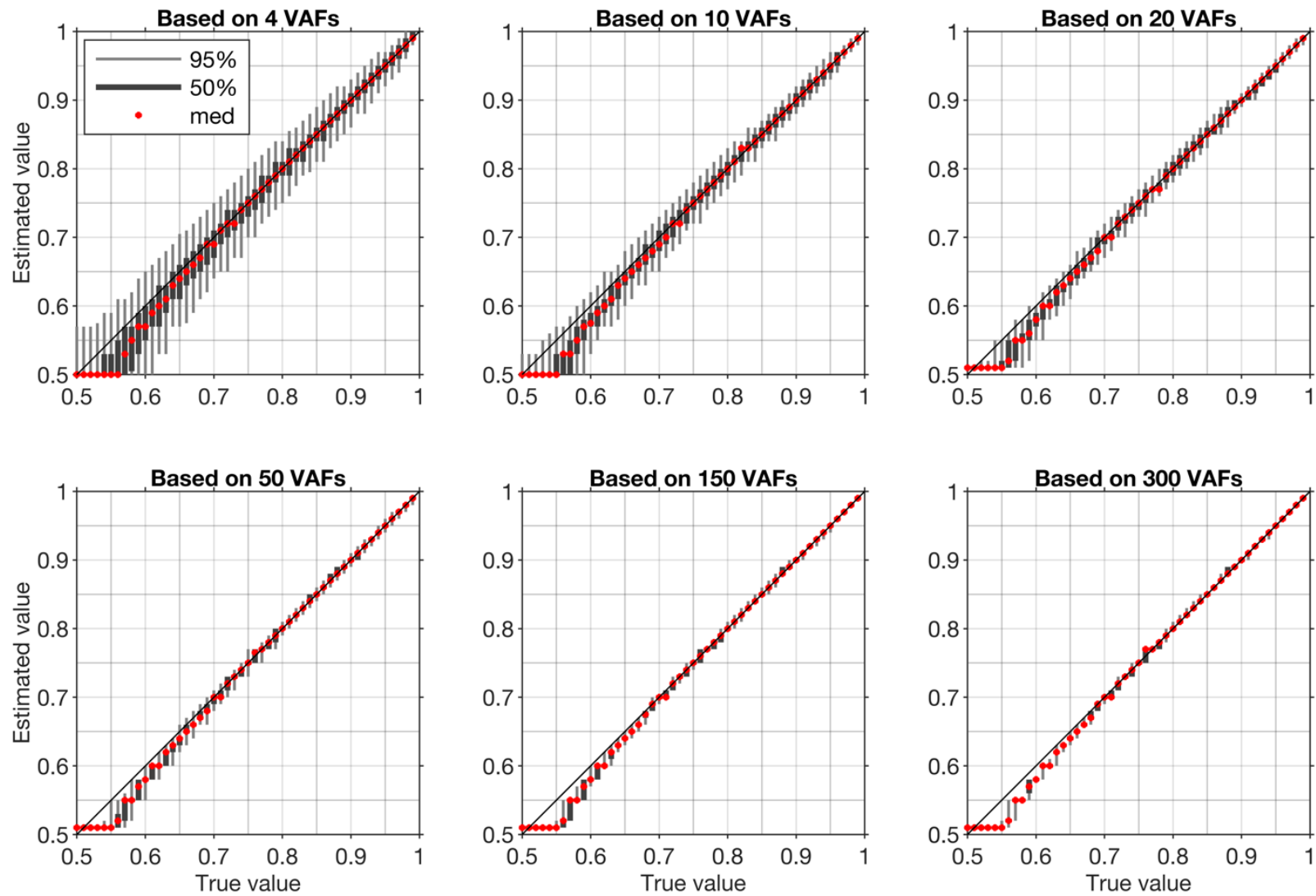

Fig. 4. Confidence intervals for artificial samples with different numbers of VAFs. Each confidence interval is based on estimation of VPR in 1000 randomly generated samples with a fixed VPR (True value). Light grey bar is 95\% confidence interval (950 samples lay within this interval), dark grey bar is 50\% confidence interval (500 samples lay within this interval), red dot is median value.

\subsubsection{V Values in mixtures of normal and tumour populations}

Since the $V_{P R}$ can take any value between 0.5 and 1 it can correspond to a single genomic event as well as any combination of genomic events in any mixture of normal and tumour populations. A mixture $v_{P R}$ value that corresponds to a combination of genomic events can be computed using the following expression:

$$
\mathrm{v}_{\mathrm{PR}}=\frac{\sum_{\mathrm{pl}=1}^{\mathrm{pl}=\mathrm{N}} \sum_{\mathrm{e}_{\mathrm{VAR}}=\{\text { events }\}} \mathrm{e}_{\mathrm{VAR}} \cdot \mathrm{p}_{\mathrm{PL}}}{\sum_{\mathrm{pl}=1}^{\mathrm{pl}=\mathrm{N}} \sum_{\mathrm{e}_{\mathrm{VAR}}=\{\text { events }\}} \mathrm{e}_{\mathrm{VAR}} \cdot \mathrm{p}_{\mathrm{PL}}+\sum_{\mathrm{pl}=1}^{\mathrm{pl}=\mathrm{N}} \sum_{\mathrm{e}_{\mathrm{REF}}=\{\text { events }\}} \mathrm{e}_{\mathrm{REF}} \cdot \mathrm{p}_{\mathrm{PL}}}
$$

Where $e_{V A R}$ and $e_{\text {REF }}$ are the multiplicities of variant and reference alleles and $\mathrm{p}_{\mathrm{PL}}$ is a proportion of one of the populations. For heterozygote sites $e_{V A R}=1$ and $e_{R E F}=1$, for deletions $e_{V A R}=0$ or $e_{R E F}=0$, for du-, tri- and 
bioRxiv preprint doi: https://doi.org/10.1101/491209; this version posted April 23 2020. The copyright holder for this preprint (which was not certified by peer review) is the author/funder, who has granted bioRxiv a license to display the preprint in perpetuity. It is made available under aCC-BY 4.0 International license.

tetraplications $\mathrm{e}_{\mathrm{VAR}}$ or $\mathrm{e}_{\mathrm{REF}}$ can be equal to 2,3 or 4 , respectively. The sum of proportions $\mathrm{p}_{\mathrm{PL}}$ over the populations is equal 1. For example, for a mixture of 1 normal $\left(N, p_{N}=0.44\right)$ and 2 tumour populations ( $(T 1$, $\mathrm{p}_{\mathrm{T} 1}=0.39$ and $\left.\mathrm{T} 2, \mathrm{p}_{\mathrm{T} 2}=0.17\right), \mathrm{T} 1$ with deletion and $\mathrm{T} 2$ with deletion the mixture $\mathrm{V}_{\mathrm{PR}}$ value can be computed as follows:

$$
\begin{aligned}
\mathrm{v}_{\mathrm{PR}} & =\frac{\mathrm{p}_{\mathrm{N}} \cdot \mathrm{B}+\mathrm{p}_{\mathrm{T} 1} \cdot \mathrm{B}+\mathrm{p}_{\mathrm{T} 2} \cdot \mathrm{B}}{\mathrm{p}_{\mathrm{N}} \cdot(\mathrm{A}+\mathrm{B})+\mathrm{p}_{\mathrm{T} 1} \cdot(0+\mathrm{B})+\mathrm{p}_{\mathrm{T} 2} \cdot(0+\mathrm{B})} \\
& =\frac{0.44 \cdot 1+0.39 \cdot 1+0.17 \cdot 1}{0.44 \cdot(1+1)+0.39 \cdot(1+0)+0.17 \cdot(1+0)}=0.694 .
\end{aligned}
$$

By comparing the $V_{P R}$ values estimated from data with possible mixture $V_{P R}$ values we propose to estimate sample purity and its clonal composition. To this end, we first generate a full set of proportions of all the population in the mixture with step (increment of) 0.01 and compute all the possible $v_{P R}$ values that each of the mixtures could produce. For step 0.01: two populations (1 tumour) give 99 proportions, three populations (2 tumours) give 4851 proportions, four populations ( 3 tumours) give 156849 proportions. The matrices with mixture $V_{P R}$ values for each proportion, vary from $2 \times 2$, for two populations with deletions, to $35 \times 35$ for four populations with all events up to tetraplications. Then, we run an exhaustive approximate search over all the matrices with mixture $V_{P R}$ values over all the proportions. The search is approximate because the estimated $V_{P R}$ values have limited accuracy and because we consider only discrete values of proportions. In the analysis we define a match between estimated and mixture $V_{P R}$ values if they differ $<0.009$. The search returns a large number of admissible mixtures that could produce the estimated $V_{P R}$ values. This process is illustrated in Fig. 5.

To represent the admissible mixtures graphically we use ternary plots. Ternary plot allows to illustrate composition of three components using just two dimensions. The composition, represented by ratios of the three components which sum to a constant, is depicted as point inside or on the edge of an equilateral triangle. If the point is on the edges, the composition has only two components. To facilitate interpretation of the ternary plots, we also plot the grid lines that are parallel to the sides of the triangle. These gridlines indicate the directions of constant ratios of the components. Along such direction the ratio of one of the components is fixed and only the other two ratios vary. Examples of visualisation of admissible mixtures on ternary plots are shown in Figs. 5 and 6. 
bioRxiv preprint doi: https://doi.org/10.1101/491209; this version posted April 23, 2020. The copyright holder for this preprint (which was not certified by peer review) is the author/funder, who has granted bioRxiv a license to display the preprint in perpetuity. It is made available under aCC-BY 4.0 International license.

Estimated $v_{P R}$ values:

\section{Proportions and matrices with mixture $\mathrm{v}_{\mathrm{PR}}$ values}
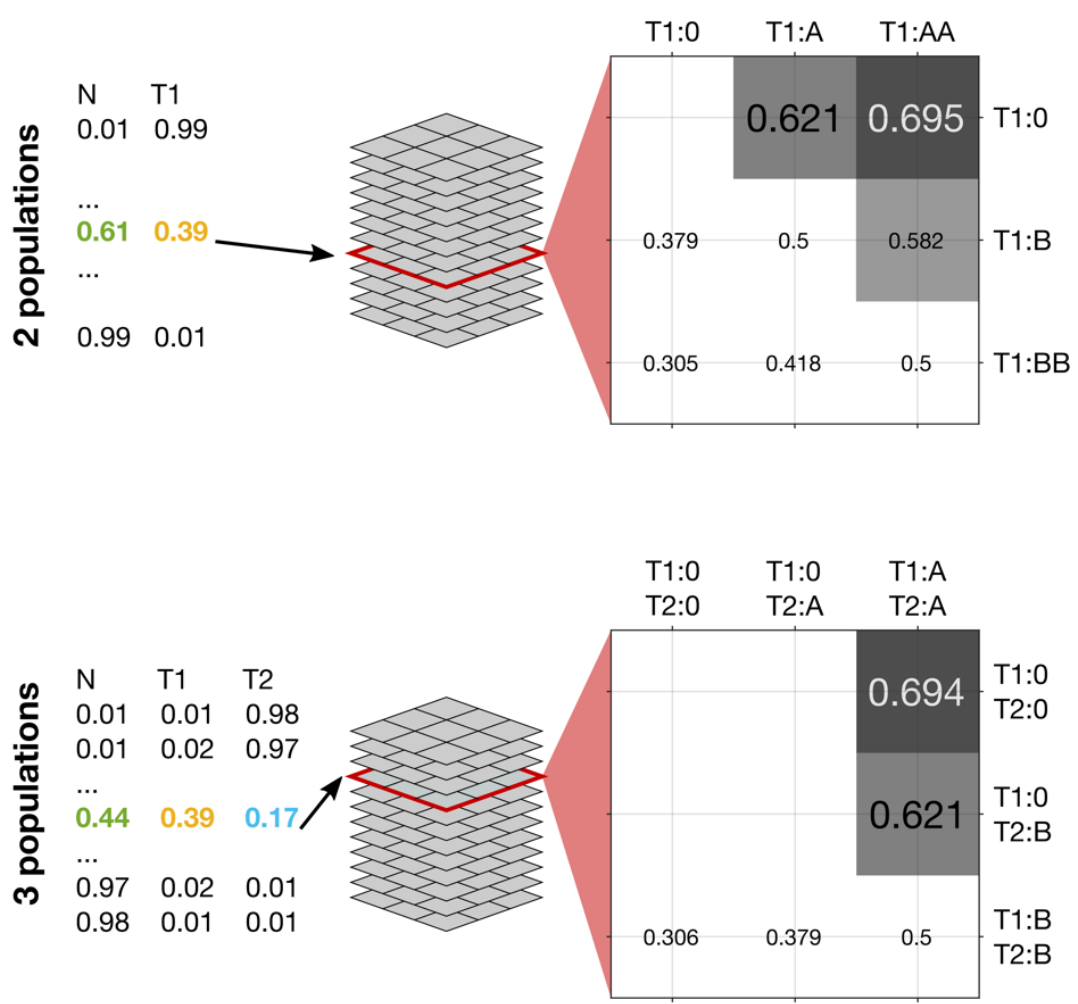

We generate list of proportions for a given number of populations. For each proportion we compute a matrix containg all the $\mathrm{V}_{\mathrm{PR}}$ values for a fixed set of the genomic events.
Examples of matrices with mixture $v_{\mathrm{PR}}$ values. The matrices contain mixture $\mathrm{V}_{\mathrm{PR}}$ values that differ less than 0.009 from ALL of the estimated $v_{P R}$ values. Meaning that a mixture used to generate the matrix is admissible.

\section{Admissible mixtures}
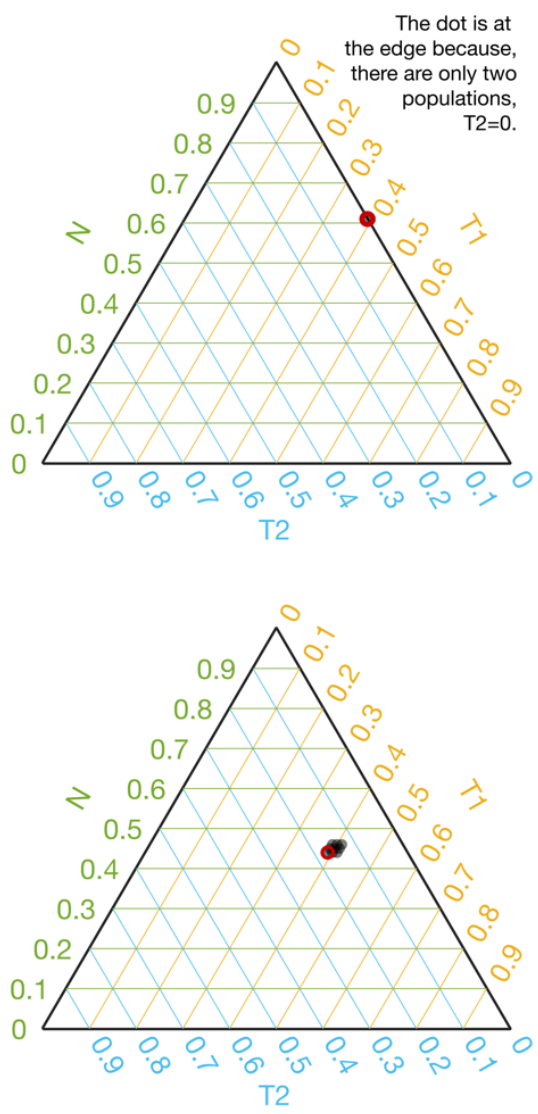

Ternary plot allow to visualise the admissible mixtures. In the ternary plot each grid line corresponds to a fix proportion of one of the populations. Values of ratios are indicated at the edges.

Fig. 5. Mixtures admissible by the $\mathbf{V}_{\mathrm{PR}}$ values estimated from data. To uncover mixtures that could produce the three estimated $V_{P R}$ values we perform an exhaustive approximate search of all the possible vpR values produced by any mixture of the populations with a given set of genetic events. In each case we generate a full set of proportions with a given step (e.g. 0.01) and compute all the possible $V_{P R}$ values that such a mixture could produce. In the illustrated cases: 2 populations (1 tumour) could produce the estimated VPR values through a deletion (estimated $\mathrm{V}_{\mathrm{PR}}=0.62$ and $\mathrm{V}_{\mathrm{PR}}=0.63$ ) and via deletion of one allele and duplication of another (estimated $\mathrm{V}_{\mathrm{PR}}=0.69$ ); 3 populations ( 2 tumours) could produce the estimated VPR values through a deletion in one of the tumour populations (estimated $V_{P R}=0.62$ and $V_{P R}=0.63$ ) and via deletion in both of the tumour populations (estimated $V_{P R}=0.69$ ). The 2 populations case admits a single mixture and the 3 populations allow 9 mixtures with similar compositions. The admissible mixtures are depicted on the ternary plots, red circle indicates solution corresponding to the presented matrix. We exclude mixture VPR values that result from deletion of both the variant and reference alleles (empty fields in the matrices).

To facilitate analysis of the admissible mixtures returned by the search procedure we introduce mixture complexity. Mixture complexity is a measure that increases with number of populations as well as with variety of genetic events. From the simplest mixture of 1 normal and 1 tumour population in which only deletions are possible to a model with 1 normal and multiple tumour populations where each can have deletions, and any level of multiplications. In practice, we set the limit at 3 tumour populations and tetraplications. Mixture complexity helps to group and visualise admissible mixtures. Mixtures with higher complexity allow more possible $V_{P R}$ values, meaning that it is easier to find the match with the estimated $V_{P R}$ values and the number of admissible mixtures increases (see Figure 6). We, further, observe that proportion of normal population, $p_{N}$, increases with a number of clonal tumour populations included in the model mixture and that, generally, $\mathrm{p}_{\mathrm{N}}$ stays constant with increasing variety of genetic events, for a fixed number of clonal tumour populations. 
bioRxiv preprint doi: https://doi.org/10.1101/491209; this version posted April 23, 2020. The copyright holder for this preprint (which was not certified by peer review) is the author/funder, who has granted bioRxiv a license to display the preprint in perpetuity. It is made available under aCC-BY 4.0 International license.

A

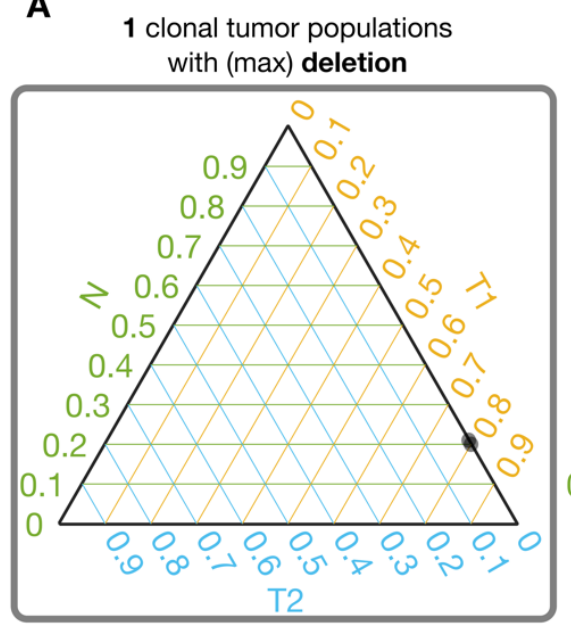

B

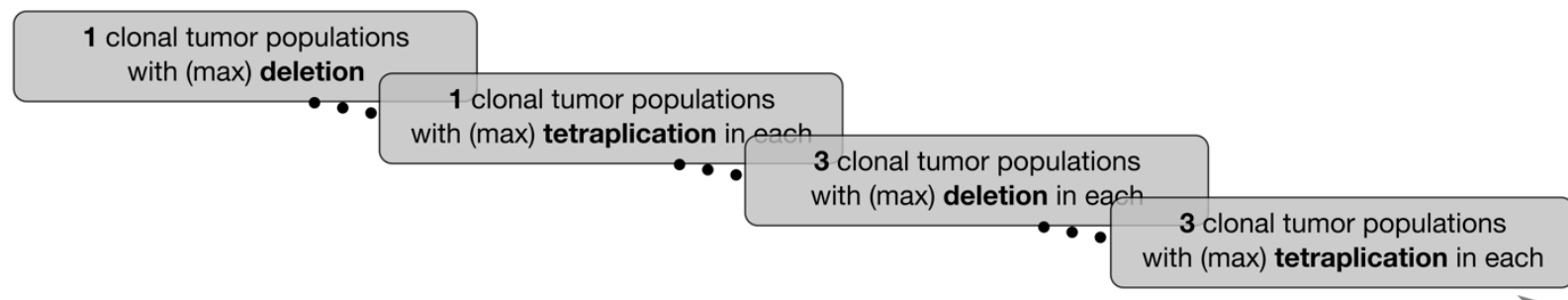

2 clonal tumor populations with (max) duplication in each

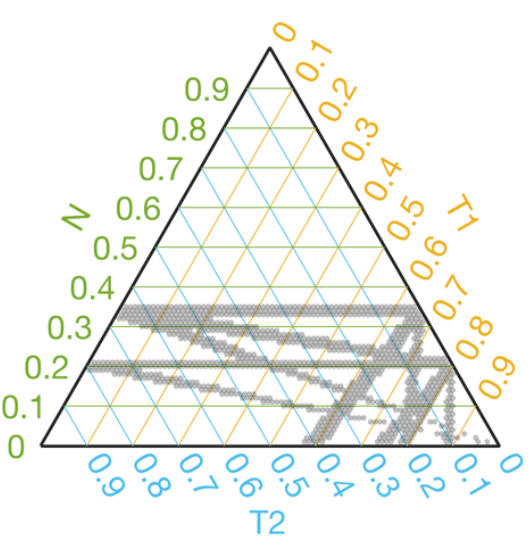

3 clonal tumor populations with (max) triplication in each

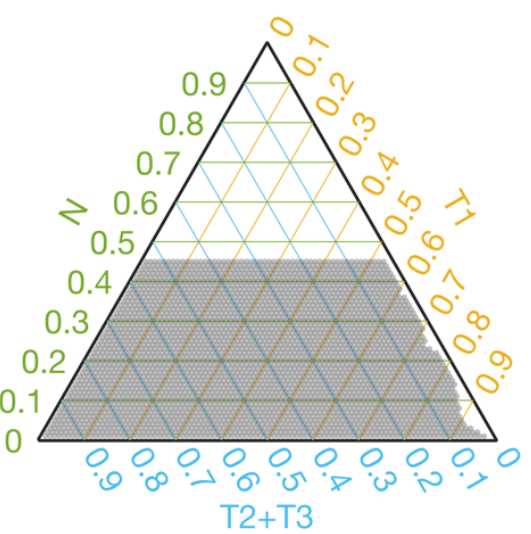

increasing mixture compexity

Fig. 6. Admissible mixtures for increasing mixture complexity. A shows admissible mixtures for 3 different mixtures with increasing complexity. The simplest mixture (mixture with the lowest number of components and the simplest set of genetic events) is show within the grey frame. On each ternary plot, the admissible mixtures are indicated by grey dots. The green axis indicates proportion of the normal population $(\mathrm{N})$, the yellow axis indicates proportion of the $1^{\text {st }}$ tumour population $(\mathrm{T} 1)$, the blue axis shows proportion of the $2^{\text {nd }}$ tumour population (T2) or sum of the $2^{\text {nd }}$ and $3^{\text {rd }}$ tumour populations $(T 2+T 3)$. B Schematic representation of increasing complexity of the mixture models. From a mixture of 1 normal and 1 tumour population in which only deletion is possible to a model with 1 normal and 3 tumour populations and each can have deletions, du-, tri- and tetraplications.

\subsection{Analysis of RNA-DNA relationships}

GetAllele is readily applicable to assess RNA-DNA relationships between normal and tumour sequencing signals derived from the same sample/individual (matched datasets). As a proof of concept, we assessed matched normal and tumour exome and transcriptome sequencing data of 72 breast carcinoma (BRCA) datasets with pre-assessed copy-number and genome admixture estimation acquired through TCGA (Supplementary Table 1). For these datasets, purity and genome admixture has been assessed using at least three of the following five approaches: ESTIMATE, ABSOLUTE, LUMP, IHC, and the Consensus Purity Estimation (CPE)(Aran, et al., 2015; Carter, et al., 2012; Katkovnik, et al., 2002; Pagès, et al., 2010; Yoshihara, et al., 2013; Zheng, et al., 2014). In addition, on the same datasets we applied THetA - a popular tool for assessing CNA and admixture from sequencing data (Oesper, et al., 2013; Oesper, et al., 2014).

\subsubsection{Segmentation results}

Segmentation of the data, based on the tumour exome signal, resulted in 2697 windows across the 72 datasets. We excluded from further analysis 294 windows where either tumour exome or transcriptome had $V_{P R}>=0.58$ but their VAF distribution could not be differentiated from the model VAF distributions with $V_{P R}=0.5$ ( $p>1$ e-5, Kolmogorov Smirnov test, equivalent to Bonferroni FWER correction for 100000 comparisons). The 294 excluded windows correspond to $4 \%$ of the data in terms of number of base pairs in the windows and $4 \%$ of all the available data points; i.e. they are short and contain only few VAF values. In the remaining 2403 windows, we systematically examined the similarity between corresponding $V_{A F} F_{T E X}$ (tumour exome), VAF (tumour transcriptome) and CNA. We obtained several distinct patterns of coordinated RNA-DNA allelic behaviour as well as correlations with CNA data. 
bioRxiv preprint doi: https://doi org/10,1101/491209; this version posted April 23, 2020. The copyright holder for this preprint (which was not certified by peer review) is the author/funder, who has granted bioRxiv a license to display the preprint in perpetuity. It is made available under aCC-BY 4.0 International license.

In $60 \%$ of all analysed windows the distributions of $\mathrm{VAF}_{\mathrm{TEX}}$ and $\mathrm{VAF}_{\mathrm{TTR}}$ were statistically concordant (had $p>1 e-5$, Kolmogorov Smirnov test), and in 40\% they were statistically discordant ( $p<1 e-5$, Kolmogorov Smirnov test). In 2 windows $V_{A F} F_{T E X}$ and $V_{A F} F_{T R}$ had the same $V_{P R}$ but had statistically different distributions $(\mathrm{p}<1 \mathrm{e}-5$, Kolmogorov Smirnov test), we consider such windows as concordant; Kolmogorov-Smirnov test is very sensitive for differences between distributions, $V_{\mathrm{PR}}$ fitting is more robust. In the vast majority of the discordant windows $V_{P R}$ of the $V_{A F} F_{T R}, V_{P R}, T R$, was higher than $V_{P R}$ of the $V_{A} F_{T E X}, V_{P R, T E X}$, (only in 21 out of 959 discordant windows $\mathrm{V}_{\mathrm{PR}, \mathrm{TR}}$ was lower than $\left.\mathrm{V}_{\mathrm{PR}, \mathrm{TEX}}\right)$.

\subsubsection{Concurrence of segmentation based on DNA and RNA}

We next analysed the concurrence between windows resulting from independent segmentations of the dataset based on the tumour exome and transcriptome signals in the datasets (2697 and 3605 windows, respectively, across all the samples). We first assessed chromosome-wise alignment of the start and end points of the windows. In $45 \%$ of the chromosomes both $\mathrm{VAF}_{\text {TEX }}$ and $\mathrm{VAF}_{\text {TTR }}$ signals produce a single window that contains the whole chromosome. In $33 \%$ of chromosomes both signals produced multiple windows. These windows are well aligned, with $90 \%$ of the break-points within $7 \%$ difference in terms of the number of data points in the chromosome (Q50=0.02\%, Q75 $=2 \%$ of data points in the chromosome). The probability of observing such an alignment by chance is smaller than $p=1 e-5$ (100,000 bootstrap samples with breaking points assigned randomly in all the individual chromosomes where both signals produced multiple windows). In $22 \%$ of the chromosome windows based on $\mathrm{VAF}_{T E X}$ and $\mathrm{VAF}_{T R R}$ the signals were positionally discordant one signal produced a single window containing whole chromosome while the other produced multiple windows.

To compare the $V_{P R}$ values in the $55 \%$ of chromosomes where at least one signal produced more than one window, we computed chromosome-wise mean absolute error (MAE) between the $\mathrm{V}_{\mathrm{PR}}$ in two sets of windows. To account for different start and end points of the windows we interpolated the $V_{P R}$ values (nearest neighbour interpolation) at each data point in the chromosome. We separately compared the $V_{P R, T E X}$ and $V_{\text {PR,TR }}$ values. The alignment in terms of MAE is very good, $\mathrm{V}_{\mathrm{PR}, \mathrm{TEX}}$ agreed perfectly in $11 \%$ of the chromosomes and had the percentiles of MAE equal to $\mathrm{Q} 50=0.012, \mathrm{Q} 75=0.022$ and $\mathrm{Q} 97.5=0.047$, while $\mathrm{V}_{\mathrm{PR}, \mathrm{TR}}$ agreed perfectly in $8 \%$ but had slightly higher percentiles of MAE Q50=0.019, Q75 $=0.034$ and Q97.5=0.07. VPR,TEX and $V_{P R, T R}$ values had $M A E=0$ simultaneously in $4 \%$ of the chromosomes. Probability of observing such values of MAE by chance is smaller than $p=1 e-3$ (1000 random assignments of $V_{P R, T E X}$ and $V_{P R, T R}$ values to windows in the 873 chromosomes where at least one signal had more than one window). It is noteworthy that MAE Q97.5<0.07 is comparable with the confidence interval of single $V_{P R}$ estimate based on 50 VAF values. In other words, both signals in a sample (Tex and Ttr) give very similar results in terms of windows' segmentation and estimated values of the $\mathrm{V}_{\mathrm{PR}}$. Albeit, segmentation of $\mathrm{VAF}_{\mathrm{TTR}}$ generates a higher number of windows. The higher number of $\mathrm{VAF}_{\mathrm{TR}}$ windows indicates that transcriptional regulation occurs at a smaller scale than allelic status changes in DNA. Figure 7 shows examples of concurrence between windows based on $\mathrm{VAF}_{\mathrm{TEX}}$ and $\mathrm{VAF}_{\mathrm{TTR}}$ signals in a positionally concordant chromosome (both signals produced multiple windows). 
bioRxiv preprint doi: https://doi.org/10.1101/491209; this version posted April 23 2020. The copyright holder for this preprint (which was not certified by peer review) is the author/funder, who has granted bioRxiv a license to display the preprint in perpetuity. It is made available under aCC-BY 4.0 International license.

A
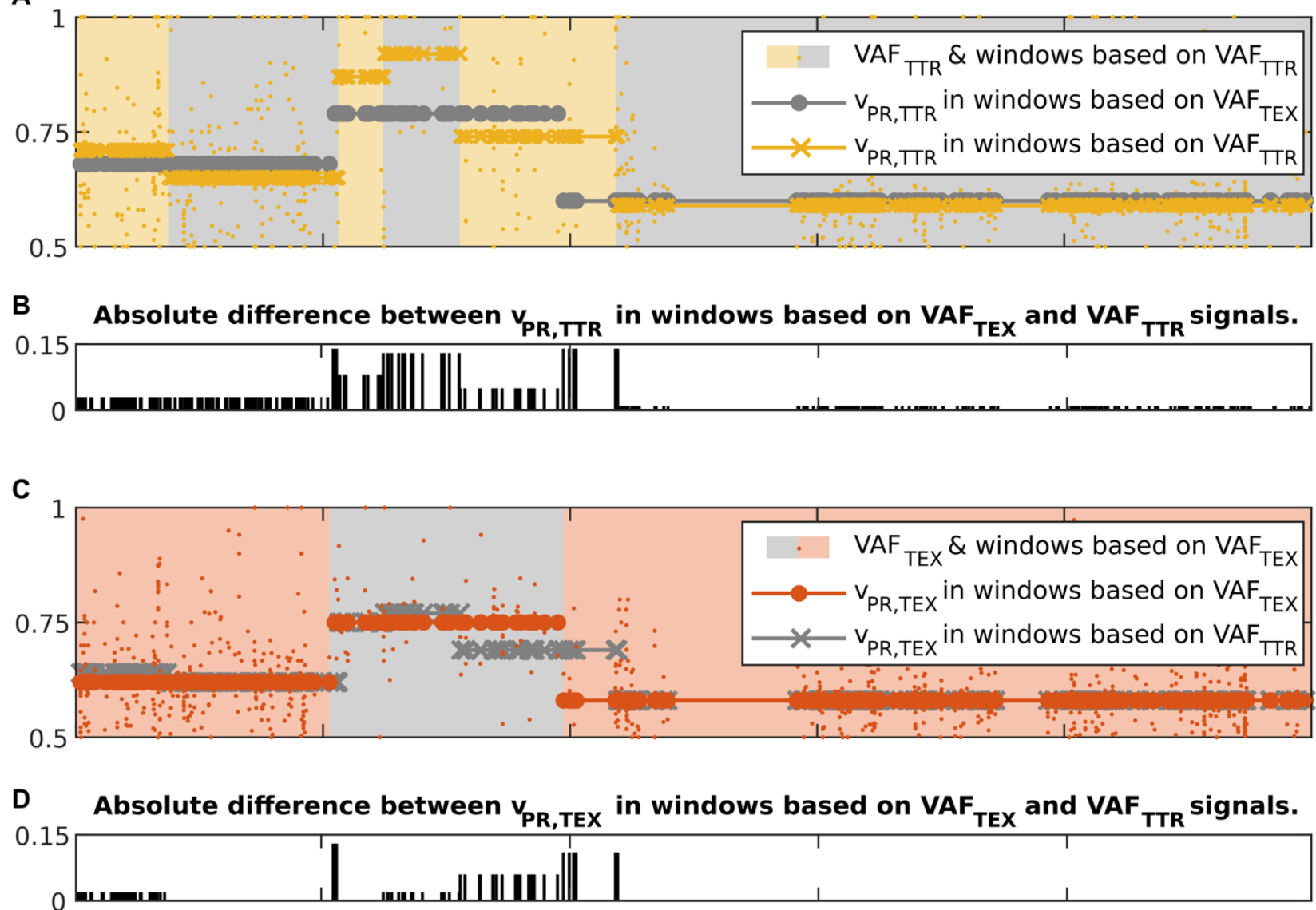

BP along a chromosome

Fig. 7. Illustration of concurrence between windows resulting from independent segmentations of the dataset based on the

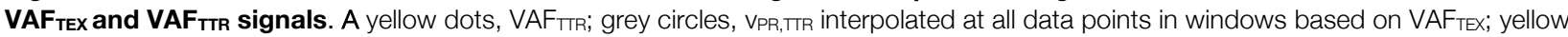
crosses, VPR,TR interpolated at all data points in windows based on VAFTR. B bar plot of the absolute difference between the VPR values in the two kinds of windows. C orange dots, VAFTEX; grey crosses, VPR,TEX interpolated at all data points in windows based on VAFTEX; orange dots VPR,TEX interpolated at all data points in windows based on VAFTTR. D bar plot of the absolute difference between the VPR values in the two kinds of windows.

\subsubsection{Correlation between $\mathrm{V}_{\mathrm{PR}}$ and CNA}

Finally, we assess the correlations between $V_{P R}$ and CNA in the individual datasets. We separately computed correlations for deletions and amplifications. In order to separate deletions and amplifications, for each data set we found $C_{N A} A_{M N}$, value of the CNA in the range -0.3 to 0.3 that had the smallest corresponding $V_{P R \text {,TEX. TO }}$ account for observed variability of the CNA values near the CNAMIN, we set the threshold for amplifications to $\mathrm{CNA}_{A}=\mathrm{CNA}_{\mathrm{MIN}}-0.05$, and for deletions we set it to $C N A_{D}=\mathrm{CNA}_{\mathrm{MIN}}+0.05$.

For $\mathrm{VAF}_{\mathrm{TEX}}$ we observed significant correlations with negative trend between $\mathrm{VPR}_{\mathrm{PR}, \mathrm{TEX}}$ and $\mathrm{CNA} \leq \mathrm{CNA}_{\mathrm{D}}$ in 57 datasets and with positive trend between $\mathrm{V}_{\mathrm{PR}, \mathrm{TEX}}$ and $\mathrm{CNA} \geq \mathrm{CNA}_{\mathrm{A}}$ in 39 datasets ( $\mathrm{p}_{\mathrm{FDR}}<0.05$, Pearson's correlation with Benjamini Hochberg multiple comparison correction for 72 samples). For $V_{A F} F_{T R}$ we observed significant correlations with negative trend between $\mathrm{V}_{\mathrm{PR}, \mathrm{TR}}$ and $\mathrm{CNA} \leq \mathrm{CNA} \mathrm{A}_{\mathrm{D}}$ in 62 datasets and with positive trend between $V_{P R, T R}$ and $C N A \geq C N A_{A}$ in 33 datasets $\left(p_{F D R}<0.05\right.$, Pearson correlation with Benjamini Hochberg correction). These correlations indicate that although $\mathrm{V}_{\mathrm{PR}, \mathrm{TEX}}$ as well as $\mathrm{V}_{\mathrm{PR}, \mathrm{TR}}$ values capture information contained in CNA, they do not differentiate between positive and negative values of the CNA. 
bioRxiv preprint doi: https://doi.org/10.1101/491209; this version posted April 23, 2020. The copyright holder for this preprint (which was not certified by peer review) is the author/funder, who has granted bioRxiv a license to display the preprint in perpetuity. It is made available under aCC-BY 4.0 International license.

A

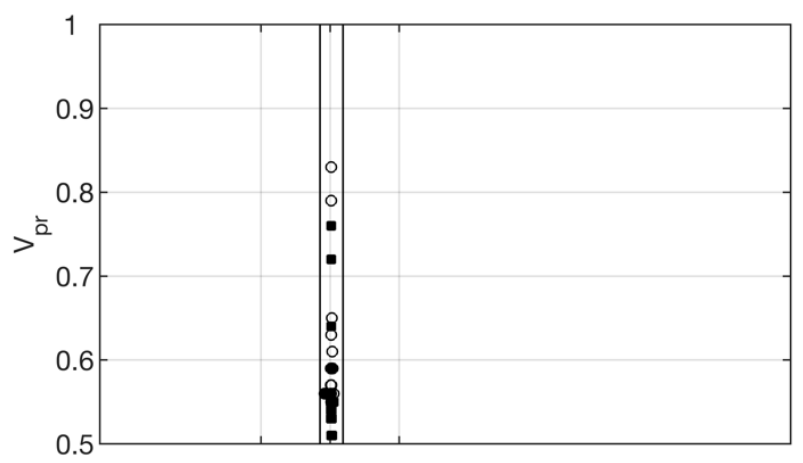

C

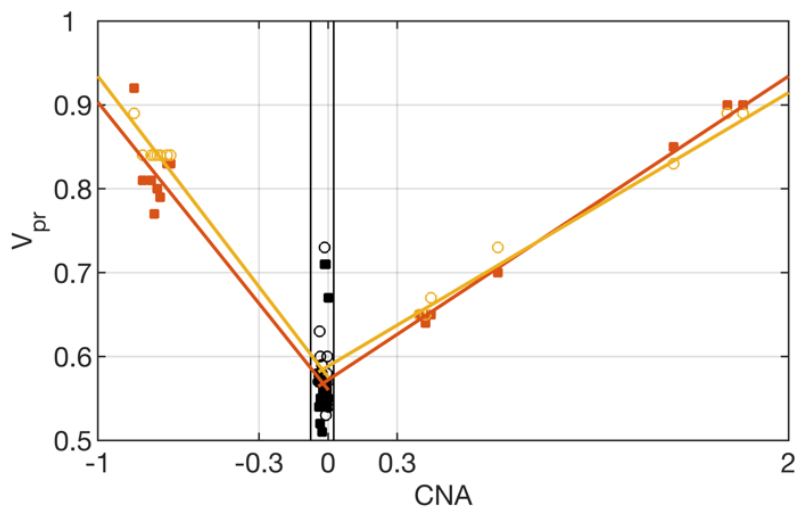

B

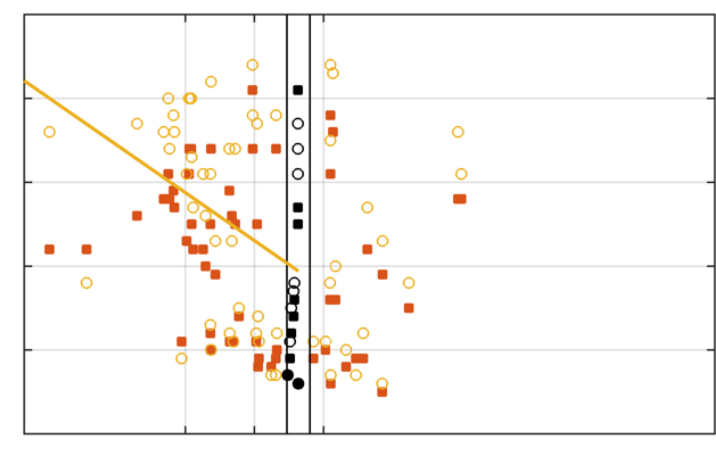

D

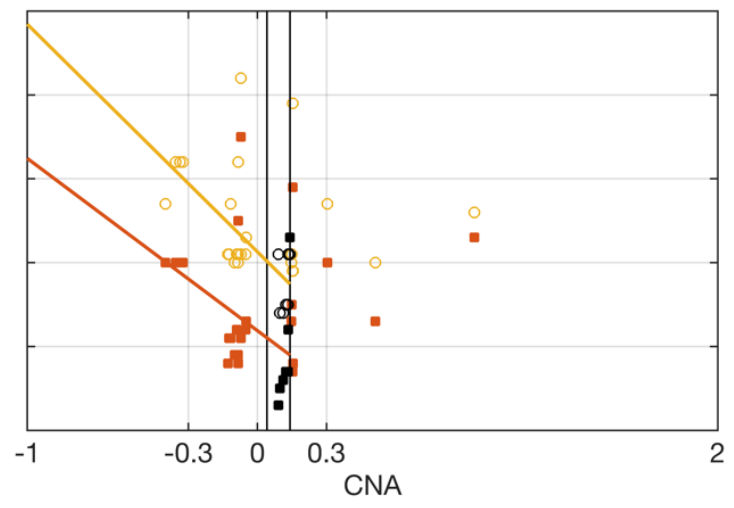

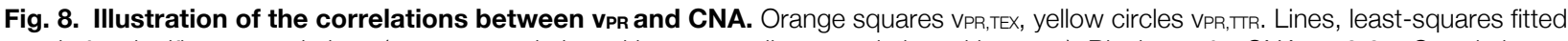
trends for significant correlations (orange correlation with VPR,TEX, yellow correlation with VPR,TRR). Black, VPR for CNAMIN \pm 0.05 . Correlations

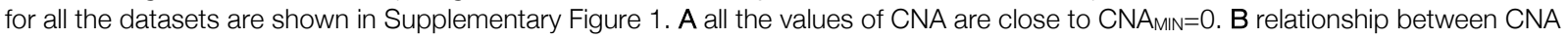
and VPR is noisy, only some correlations are statistically significant. $\mathbf{C}$ all the correlations are statistically significant, VPR,TRR values (circles) follow closely the $V_{P R}$,TEX (squares) indicating concordance of the VAFTEX and VAFTR distributions. D only correlations for CNA $\leq$ CNA ${ }_{D}$ are statistically significant.

Figure 8 shows four typical patterns of correlation between the CNA and $V_{P R}$ values observed in the data. In Figure $8 \mathrm{~A}$ all the values of CNA are close to $\mathrm{CNA}_{\mathrm{MIN}}$. In Figure $8 \mathrm{~B}$ the relationship between CNA and $\mathrm{V}_{\mathrm{PR}}$ is noisy, only correlation between $V_{P R, T R}$ and $C N A \leq C N A_{D}$ are statistically significant $\left(r_{T E X, C N A, D}=-0.29, p_{F D R}=\right.$ $0.063 ; r_{T E X, C N A, D}=-0.38, p_{\text {FDR }}=0.012 ; r_{T E X, C N A, A}=0.14, p_{F D R}=0.58 ; r_{T E X, C N A, A}=0.19, p_{F D R}=0.47 ;$ Pearson's correlation with Benjamini Hochberg multiple comparison correction for 72 samples). In Figure $8 \mathrm{C}$ all the correlations are statistically significant, $\mathrm{VPR}_{\mathrm{PR}, \mathrm{TR}}$ values (circles) follow closely the $\mathrm{VPR}_{\mathrm{PR}, \mathrm{TEX}}$ (squares) indicating that in most of the windows distributions of the $V A F_{T E X}$ and $V A F_{T R}$ are concordant $\left(r_{T E X, C N A, D}=-0.91, p_{F D R}<1 e-10\right.$; $\left.r_{T E X, C N A, D}=-0.96, p_{F D R}<1 e-10 ; r_{T E X, C N A, A}=0.92, p_{F D R}<1 e-10 ; r_{T E X, C N A, A}=0.95, p_{F D R}<1 e-10\right)$. In Figure 8D correlations between $V_{P R, T E X}, V_{P R}, T R R$ and $C N A \leq C N A_{D}$ are statistically significant, but there is a big difference (with median of 0.18 ) between $\mathrm{V}_{\mathrm{PR}, \mathrm{TEX}}$ and $\mathrm{V}_{\mathrm{PR}, \mathrm{TR}}$ values, indicating that in most of the windows the distributions of the $\mathrm{VAF}_{\mathrm{TEX}}$ and $\mathrm{VAF}_{\mathrm{TTR}}$ in this dataset are discordant $\left(\mathrm{r}_{\mathrm{TEX}, \mathrm{CNA}, \mathrm{D}}=-0.44, \mathrm{p}_{\mathrm{FDR}}=0.047 ; \mathrm{r}_{\mathrm{TEX}, \mathrm{CNA}, \mathrm{D}}=\right.$ -0.64, $\left.p_{F D R}=0.0017 ; r_{T E X, C N A, A}=0.44 p_{F D R}=0.16 ; r_{T E X, C N A, A}=0.28, p_{F D R}=0.41\right)$. In many of the datasets we observe that the $\mathrm{V}_{\mathrm{PR}, T R}$ values are higher than the corresponding $\mathrm{V}_{\mathrm{PR}, \mathrm{TEX}}$ Values (median $\mathrm{V}_{\mathrm{PR}, \mathrm{TR}}-\mathrm{V}_{\mathrm{PR}, \mathrm{TEX}}=0.03$ ). Correlations between $V_{P R}$ and CNA in all datasets are shown in the Supplementary Figure 1.

\section{3 $\quad V_{\mathrm{PR}}$ based purity estimation}

To demonstrate a practical application of estimating the admissible mixtures by means of comparing the estimated $V_{P R}, T E X$ values with the mixture $V_{P R}$ values we next use them to estimate purity of the samples. To this end we compared the $V_{P R}$ based purity (VBP) estimates with ESTIMATE, ABSOLUTE, LUMP, IHC, and the Consensus Purity Estimation (CPE)(Aran, et al., 2015; Carter, et al., 2012; Katkovnik, et al., 2002; Pagès, et al., 2010; Yoshihara, et al., 2013; Zheng, et al., 2014).

To obtain the VBP estimate we used $V_{P R, T E X}$ values. We, first, selected the $V_{P R, T E X}$ values that: 1 . are estimated with high confidence, i.e. are based on at least 50 data points; 2 . are most likely heterozygous in normal exome, i.e., have a corresponding $V_{P R}$ value in normal exome $V_{P R, N E X}<0.59$; 3. most likely have $V_{P R, T E X}>$ 
bioRxiv preprint doi: https://doi org/10.1101/491209; this version posted April 23, 2020. The copyright holder for this preprint (which was not certified by peer review) is the author/funder, who has granted bioRxiv a license to display the preprint in perpetuity. It is made available under aCC-BY 4.0 International license.

0.5 , i.e., their $p$-value for comparison with $v_{P R, T E X}=0.5$ is very small $p<1 e-5$ (Kolmogorov-Smirnov test). Next, we used the selected VPR,TEX values to find all admissible mixtures (with 1 to 3 tumour populations and allowing for all events, from deletions to tetraplications). To estimate the VBP, out of all the admissible mixtures we chose these with lowest mixture complexity and among these mixtures we take one with the highest $\mathrm{p}_{\mathrm{N}}$ (proportion of the normal population). The VBP, percentage of tumour populations in the sample, is then given as 1- $\mathrm{p}_{\mathrm{N}}$. Such approach provides rather conservative estimates of VBP (the smallest 1- $\mathrm{p}_{\mathrm{N}}$ ). However,

GetAllele can be extended to offer alternative methods of employing the admissible mixtures to estimate VBP. Development, analysis and comparison of alternative VBP estimation methods is beyond scope of the current paper.

Figure 9A shows violin plots of all considered $1-p_{N}$ values and $(x)$ indicates the smallest value taken as a VBP estimate. In two of the datasets we could not estimate the purity due to lack of suitable VPR,TEX values. The VBP estimates shows the best agreement with ABSOLUTE method $(r=0.76, p<3.4 \mathrm{e}-14$, Pearson's correlation, Fig. 9B2). We suppose that this is because the ABSOLUTE method is based on copy number distributions, and our analysis (Section 2.2.3) revealed high correlations between the CNAs and VPR values. Similarly to the ABSOLUTE method, VBP estimates are generally lower than the other purity estimates (ESTIMATE, LUMP, IHC, CPE); see Fig. 9B1-B5.

The approach presented in this section differs from other methods for inferring genomic mixture composition (e.g. SciClone (Miller, et al., 2014), PyClone (Roth, et al., 2014) or TPES (Locallo, et al., 2019)) in that it is based on changes of the alleles multiplicity estimated along continuous multi-SNV genomic regions. The $V_{P R}$ values used to estimate admissible mixtures are based on windows with at least $50 \mathrm{VAF}$ values which extend over millions of base pairs. In this way, the presented method is complementary to the SciClone and PyClone methods that use VAFs of somatic mutations. 
A

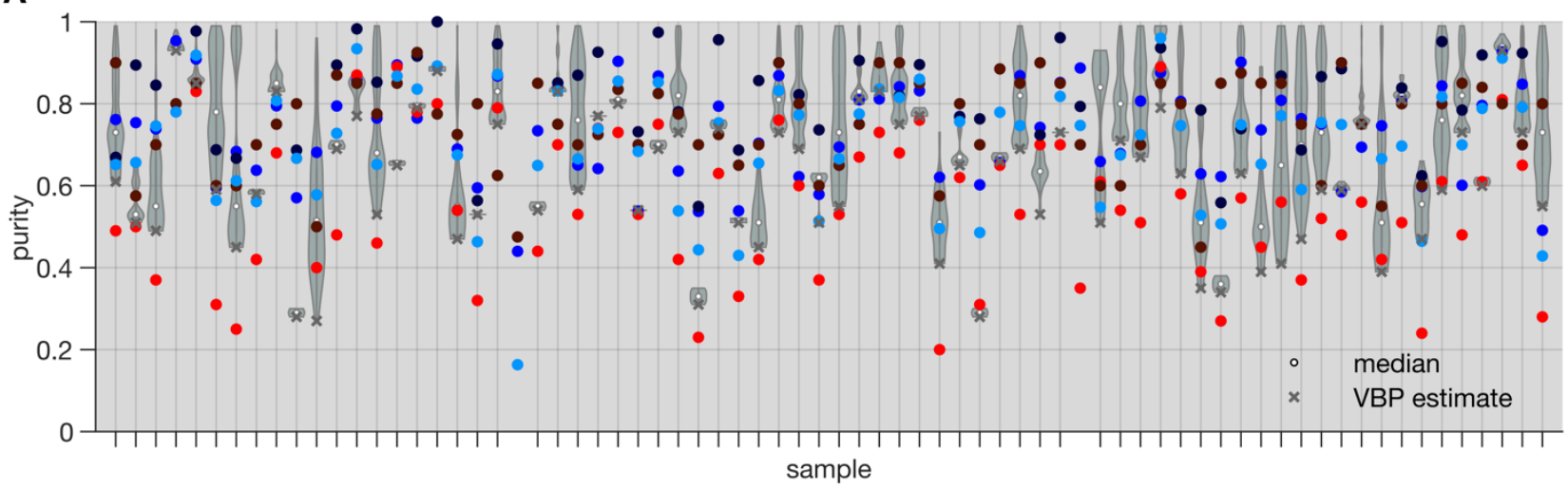

B1

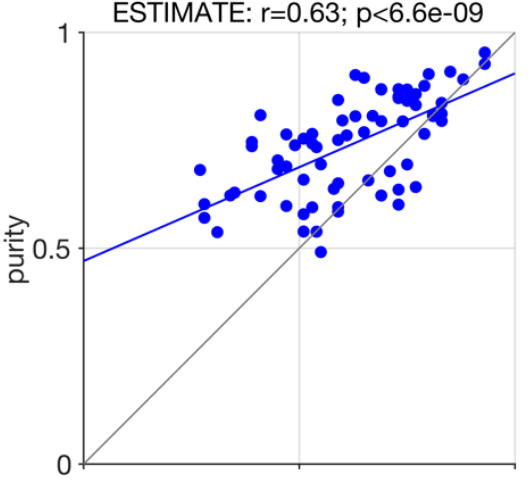

B4

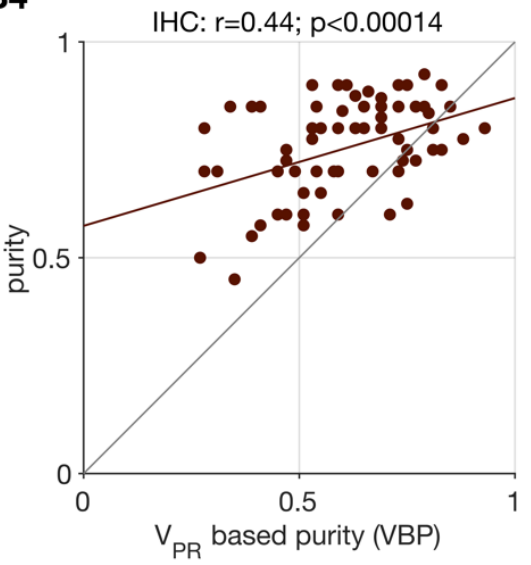

B2

ABSOLUTE: $r=0.76 ; p<3.4 e-14$

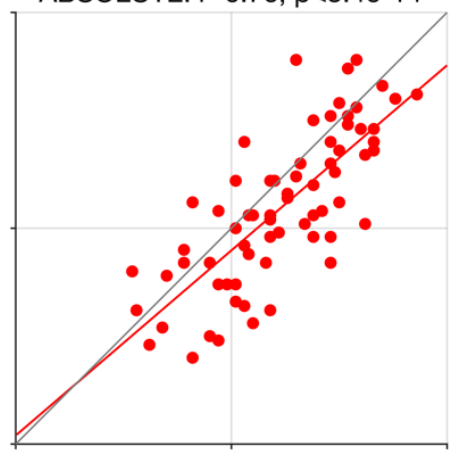

B5

CPE: $r=0.72 ; p<2.8 e-12$

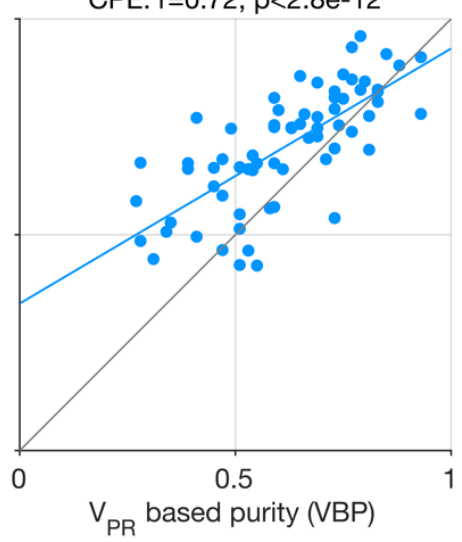

B3

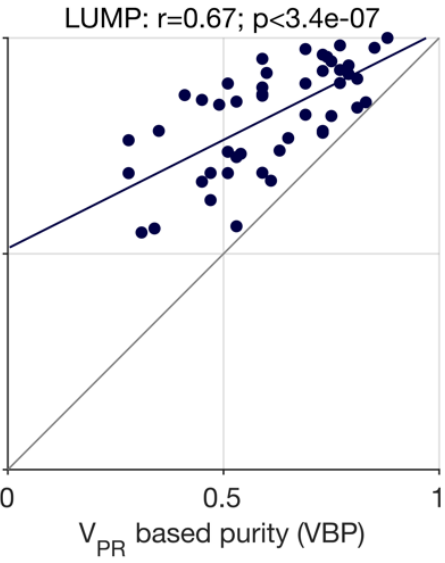

C

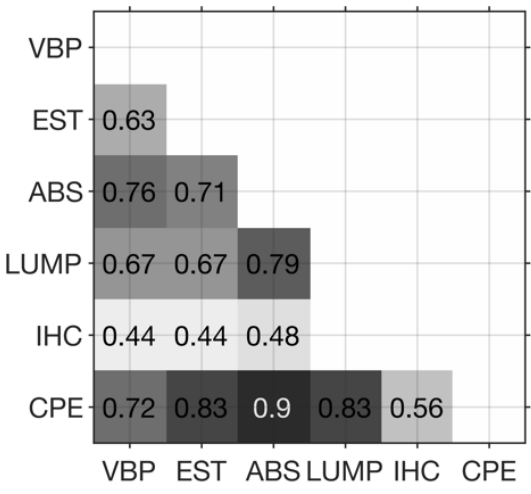

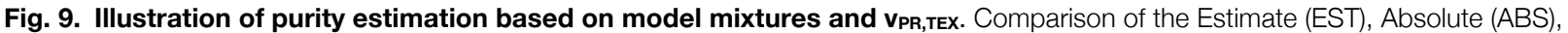
LUMP, IHC, and the consensus purity estimate (CPE) methods with VPR based purity (VBP). A violin plots show distributions of purity based on all the admissible proportions of the normal population $(\mathrm{x}$ ) indicates the lowest value selected as the most conservative estimate; colours corresponding to the different methods are indicated in B1-5. B1-5 Correlation of the VBP with individual methods; coloured line indicates best fit linear trend. C. Matrix showing significant $p<0.05$ Pearson's correlation coefficients between all tested methods.

\section{Discussion}

Potential for integrative analysis of RNA and DNA sequence data is facilitated by the growing availability of RNA and DNA sequencing datasets and by the technological advances now enabling simultaneous RNA and DNA sequencing from the same source (Macaulay, et al., 2016; Reuter, et al., 2016; The, et al., 2012). However, integrative RNA and DNA analyses are challenged by limited compatibility between RNA and DNA datasets and high technical variance of the sequencing-produced signals. Our approach - GeTallele addresses the compatibility by restricting the analyses to confidently co-covered DNA and RNA regions, and the high variability - through quantification of differences between the DNA and RNA signals.

In contrast to other methods based on statistical modelling, GeTallele is based on a mechanistic model of VAF distributions that depends on the distribution of total reads (extracted from data) and the VPR parameter. The brute-force simplicity and transparency of the presented methodology is one of its biggest advantages. 
bioRxiv preprint doi: https://doi.org/10.1101/491209; this version posted April 23, 2020. The copyright holder for this preprint (which was not certified by peer review) is the author/funder, who has granted bioRxiv a license to display the preprint in perpetuity. It is made available under aCC-BY 4.0 International license.

Additionally, in contrast to other methods, to analyse sequencing data GeTallele uses data segments that include multiple adjacent SNVs. The proposed mechanistic model indicates that due to probabilistic nature of the reads that estimates of genomic events based on continuous multi-SNV regions are intrinsically more robust than estimates based on a single SNV. Finally, GeTallele offers a unified pipeline to evaluate and visualise DNA-RNA whereas many of the other methods cited herein are used in conjunction with each other to achieve a similar result.

Using GeTallele, we detected several relationships between DNA-RNA allele frequencies and biological processes. First, in chromosomes affected by deletions and amplifications, $V F_{\mathrm{RNA}}$ and $\mathrm{VAF}_{\mathrm{DNA}}$ showed highly concordant breakpoint calls. This indicates that $\mathrm{VAF}_{\mathrm{RNA}}$ alone can serve as preliminary indicator for deletions and amplifications, which can facilitate the applications of RNA-sequencing analysis on the large and constantly growing collections of transcriptome sequencing data. Second, we showcased that VPR,TEX can be used to estimate sample composition (in terms of proportions of normal and tumour populations) and as a result its purity. Once the mixture composition is decided by the user, GeTallele allow to further interrogate genetic events in each population at a specific data segment.

\section{Conclusions}

Based on our results, variant probability VPR can serve as a dependable indicator to assess gene and chromosomal allele asymmetries and to aid calls of genomic events in matched sequencing RNA and DNA datasets. Methods for estimating and analysing $V_{P R}$ values are implemented in a GeTallele toolbox. GeTallele provides a singular suit of functions for integrative analysis, statistical assessment and visualization of the observed patterns at desired resolution, including chromosome, gene, or custom genome region.

\section{Methods}

\subsection{Samples}

The GeTallele was developed using sequencing datasets from paired normal and tumour tissue obtained from 72 female patients with breast invasive carcinoma (BRCA) from The Cancer Genome Atlas (TCGA). Each of the 72 datasets contains four matched sequencing datasets: normal exome (Nex), normal transcriptome (Ntr), tumour exome (Tex), and tumour transcriptome (Ttr). In addition, we required each tumour sample to have at least three of the following five purity estimates - Estimate, Absolute, LUMP, IHC, and the consensus purity estimate (CPE), (Supplementary Table 1). Finally, each sample was required to have CNA estimation (genomic segment means based on Genome-Wide-SNPv6 hybridization array) (Aran, et al., 2015; Carter, et al., 2012; Katkovnik, et al., 2002; Pagès, et al., 2010; Yoshihara, et al., 2013; Zheng, et al., 2014).

\subsection{Data processing}

All datasets were generated through paired-end sequencing on an Illumina HiSeq platform. The human genome reference (hg38)-aligned sequencing reads (Binary Alignment Maps, .bams) were downloaded from the Genomic Data Commons Data Portal (https://portal.gdc.cancer.gov/) and processed downstream through an in-house pipeline. After variant call (Li, 2011), the RNA and DNA alignments, together with the variant lists were processed through the read count module of the package RNA2DNAlign (Movassagh, et al., 2016), to produce variant and reference sequencing read counts for all the variant positions in all four sequencing signals (normal exome, normal transcriptome, tumour exome and tumour transcriptome). Selected read count assessments were visually examined using Integrative Genomics Viewer (Thorvaldsdóttir, et al., 2013).

\subsection{Data segmentation}

To analyse variant allele frequencies (VAF) at genome-wide level, GeTallele first divides the VAF dataset into a set of non-overlapping windows along the chromosomes. Segmentation of the dataset into windows is based on a sequencing signal chosen out of all the available datasets in the aggregated aligned VAF dataset (one out of four in the presented analysis).

To partition the data into the windows GeTallele uses a parametric global method, which detects the breakpoints in the signals using its mean, as implemented in the Matlab function findchangepts (Killick, et al., 2012; Lavielle, 2005) or in R (Killick and Eckley, 2014). In each window, the VAF values of the chosen signal have a mean that is different from the mean in the adjacent windows. Sensitivity of breakpoint detection can be controlled using parameter MinThreshold, in the presented analysis it was set to 0.2. For 
bioRxiv preprint doi: https://doi.org/10.1101/491209; this version posted April 23, 2020. The copyright holder for this preprint (which was not certified by peer review) is the author/funder, who has granted bioRxiv a license to display the preprint in perpetuity. It is made available under aCC-BY 4.0 International license.

segmentation and analysis (without loss of generality) we transform all the original VAF values to VAF=|VAF$0.5 \mid+0.5$.

\subsection{Statistics}

To test statistical significance, GeTallele uses parametric and non-parametric methods and statistical tests (Corder and Foreman, 2014; Hollander, et al., 2013). Namely, to compare distributions of the variant allele frequencies (VAF) we use Kolmogorov-Smirnov test (examples of VAF distributions are depicted in Figs. 2 and 3). To study concurrence of windows, we use permutation/ bootstrap tests. To test relations between $v_{P R}$ and copy number alterations (CNA) we use Pearson's correlation coefficient.

To account for multiple comparisons, we set the probability for rejecting the null hypothesis at $p<1 e-5$, which corresponds to Bonferroni (Dunn, 1961) family-wise error rate (FWER) correction against 100000 comparisons. We use a fixed value, rather than other approaches, to ensure better consistency and reproducibility of the results. Alternatively, we apply Benjamini and Hochberg (Benjamini and Hochberg, 1995) false discovery rate (FDR) correction with a probability of accepting false positive results $p_{F D R}<0.05$. We specify the method used in the text when reporting the results.

\section{List of abbreviations}

BRCA - breast invasive carcinoma,

CDF - cumulative distribution function,

CNA - copy number alterations,

$C N A_{D}$ - copy number alterations corresponding to deletions (see section 2.2.3),

$\mathrm{CNA}_{A}-$ copy number alterations corresponding to amplifications (see section 2.2.3),

CPE - consensus purity estimate,

DNA - genome,

EMD - earth mover's distance

FWER - family-wise error rate,

FDR - false discovery rate,

MEA - mean absolute error

Nex - normal exome,

Ntr - normal transcriptome,

$r_{T E X, C N A, D}-$ Pearson's correlation coefficient between $V_{P R, T E X}$ and CNA

$r_{T E X, C N A, A}$ - Pearson's correlation coefficient between $V_{P R, T E X}$ and CNA

$r_{T T R, C N A, D}-$ Pearson's correlation coefficient between $V_{P R, T T R}$ and CNA $A_{D}$

$r_{T T R, C N A, A}$ - Pearson's correlation coefficient between $V_{P R, T R R}$ and CNA

$\mathrm{P}_{\mathrm{FDR}}-\mathrm{p}$-value after multiple comparisons Benjamini and Hochberg false discovery rate correction,

PDF - probability density function,

QN (e.g. Q50) - N-th percentile

RNA - transcriptome

SNV - single-nucleotide variant

TCGA - the cancer genome atlas,

Tex - tumour exome,

Ttr - tumour transcriptome,

$\mathrm{VAF}$ - variant allele frequency,

$\mathrm{VAF}_{\mathrm{TEX}}$ - variant allele frequency in tumour exome sequence,

$V_{A T R}$ - variant allele frequency in tumour transcriptome sequence,

VBP - $V_{P R}$ based purity,

$V_{P R}-$ variant probability,

$V_{\mathrm{PR}, \mathrm{TEX}}$ - variant probability estimated from tumour exome sequence,

$V_{\mathrm{PR}, \mathrm{TTR}}$ - variant probability estimated from tumour transcriptome sequence,

\section{Declarations}

- Ethics approval and consent to participate: Not applicable

- Consent for publication: Not applicable

- $\quad$ Availability of data and materials: The datasets used and/or analysed during the current study are available from the corresponding author on reasonable request. GeTallele is implemented as a Matlab 
bioRxiv preprint doi: https://doi.org/10.1101/491209; this version posted April 23, 2020. The copyright holder for this preprint (which was not certified by peer review) is the author/funder, who has granted bioRxiv a license to display the preprint in perpetuity. It is made available under aCC-BY 4.0 International license.

toolbox available at: https://github.com/SlowinskiPiotr/GeTallele. We are working on R implementation of GeTallele.

- Competing interests: The authors declare that they have no competing interests.

- Funding: This work was supported by McCormick Genomic and Proteomic Center (MGPC), The George Washington University; [MGPC_PG2018 to AH]. Work of PS was generously supported by the Wellcome Trust Institutional Strategic Support Award [204909/Z/16/Z]. KTA gratefully acknowledges the financial support of the EPSRC via grant EP/N014391/1.

- Authors' contributions: PS, ML, PR, NA, LFS, CM, KTA, AH conception and design of the work; ML data acquisition; PS data analysis; PS, ML, PR, LFS, KTA, AH interpretation of data; PS the creation of new software used in the work; PS, ML, PR, LFS, KTA, AH have drafted the work or substantively revised it; all authors approved the submitted version. All authors agreed both to be personally accountable for the author's own contributions and to ensure that questions related to the accuracy or integrity of any part of the work, even ones in which the author was not personally involved, are appropriately investigated, resolved, and the resolution documented in the literature.

- Acknowledgements: Not applicable

\section{References}

Aran, D., Sirota, M. and Butte, A.J. Systematic pan-cancer analysis of tumour purity. Nature communications 2015;6:8971.

Benjamini, Y. and Hochberg, Y. Controlling the False Discovery Rate - a Practical and Powerful Approach to Multiple Testing. J Roy Stat Soc B Met 1995;57(1):289-300.

Carter, S.L., et al. Absolute quantification of somatic DNA alterations in human cancer. Nature biotechnology 2012;30(5):413-421.

Corder, G.W. and Foreman, D.I. Nonparametric Statistics. John Wiley \& Sons; 2014.

Dunn, O.J. Multiple Comparisons among Means. J Am Stat Assoc 1961;56(293):52-64.

Ferreira, E., Shaw, D.M. and Oddo, S. Identification of learning-induced changes in protein networks in the hippocampi of a mouse model of Alzheimer's disease. Translational psychiatry 2016;6(7):e849.

$\mathrm{Ha}$, G., et al. Integrative analysis of genome-wide loss of heterozygosity and monoallelic expression at nucleotide resolution reveals disrupted pathways in triple-negative breast cancer. Genome research 2012;22(10):1995-2007.

Han, L., et al. Alternative applications for distinct RNA sequencing strategies. Briefings in bioinformatics 2015;16(4):629-639.

Hardy, G.H., et al. An introduction to the theory of numbers. Oxford ; New York: Oxford University Press; 2008.

Hollander, M., Wolfe, D.A. and Chicken, E. Nonparametric Statistical Methods. John Wiley \& Sons; 2013.

Kantorovich, L.V. and Rubinstein, G.S. On a space of completely additive functions. Vestnik Leningrad. Univ 1958;13(7):52-59.

Katkovnik, V., Kgiazarian, K. and Astola, J. Adaptive window size image de-noising based on intersection of confidence intervals (ICI) rule. J Math Imaging Vis 2002;16(3):223-235.

Kendall, M.G. A new measure of rank correlation. Biometrika 1938;30(1-2):81-93.

Killick, R. and Eckley I.A. changepoint: An R Package for Changepoint Analysis. Journal of Statistical Software, 2014;58(3):1-19. http://www.jstatsoft.org/v58/i03/.

Killick, R., Fearnhead, P. and Eckley, I.A. Optimal Detection of Changepoints With a Linear Computational Cost. J Am Stat Assoc 2012;107(500):1590-1598.

Kowalski, C.J. On the Effects of Non-Normality on the Distribution of the Sample Product-Moment Correlation Coefficient. Journal of the Royal Statistical Society. Series C (Applied Statistics) 1972;21(1):1-12.

Lavielle, M. Using penalized contrasts for the change-point problem. Signal Process 2005;85(8):1501-1510.

Levina, E. and Bickel, P. The Earth Mover's distance is the Mallows distance: some insights from statistics. In, IEEE International Conference on Computer Vision

2001. p. 251-256.

$\mathrm{Li}, \mathrm{H}$. A statistical framework for SNP calling, mutation discovery, association mapping and population genetical parameter estimation from sequencing data. Bioinformatics 2011;27(21):2987-2993.

Li, H., et al. The Sequence Alignment/Map format and SAMtools. Bioinformatics 2009;25(16):2078-2079.

Locallo, A., et al. TPES: tumor purity estimation from SNVs. Bioinformatics 2019;35(21):4433-4435

Macaulay, I.C., et al. Separation and parallel sequencing of the genomes and transcriptomes of single cells using G\&T-seq. Nature protocols 2016;11(11):2081-2103.

Miller CA, White BS, Dees ND, Griffith M, Welch JS, et al. SciClone: Inferring Clonal Architecture and Tracking the Spatial and Temporal Patterns of Tumor Evolution. PLOS Computational Biology 2014;10(8):e1003665. https://doi.org/10.1371/journal.pcbi.1003665

Morin, R.D., et al. Mutational and structural analysis of diffuse large B-cell lymphoma using whole-genome sequencing. Blood 2013;122(7):1256-1265

Movassagh, M., et al. RNA2DNAlign: nucleotide resolution allele asymmetries through quantitative assessment of RNA and DNA paired sequencing data. Nucleic acids research 2016;44(22):e161.

Newson, R. Parameters behind "nonparametric" statistics: Kendall's tau,Somers' D and median differences. Stata Journal 2002;2(1):4564.

Oesper, L., Mahmoody, A. and Raphael, B.J. THetA: inferring intra-tumor heterogeneity from high-throughput DNA sequencing data. Genome biology 2013;14(7):R80.

Oesper, L., Satas, G. and Raphael, B.J. Quantifying tumor heterogeneity in whole-genome and whole-exome sequencing data. Bioinformatics (Oxford, England) 2014;30(24):3532-3540.

Pagès, F., et al. Immune infiltration in human tumors: a prognostic factor that should not be ignored. Oncogene 2010;29(8):1093-1102. 
bioRxiv preprint doi: https://doi.org/10.1101/491209; this version posted April 23, 2020. The copyright holder for this preprint (which was not certified by peer review) is the author/funder, who has granted bioRxiv a license to display the preprint in perpetuity. It is made available under aCC-BY 4.0 International license.

Reuter, J.A., et al. Simul-seq: combined DNA and RNA sequencing for whole-genome and transcriptome profiling. Nature methods 2016;13(11):953-958.

Roth, A., Khattra, J., Yap, D. et al. PyClone: statistical inference of clonal population structure in cancer. Nat Methods 2014; 11, 396398. https://doi.org/10.1038/nmeth.2883

Shah, S.P., et al. The clonal and mutational evolution spectrum of primary triple-negative breast cancers. Nature 2012;486(7403):395399

Shi, L., et al. Long-read sequencing and de novo assembly of a Chinese genome. Nature communications 2016;7:12065.

Shlien, A., et al. Direct Transcriptional Consequences of Somatic Mutation in Breast Cancer. Cell reports 2016;16(7):2032-2046.

The, E.P.C., et al. An integrated encyclopedia of DNA elements in the human genome. Nature 2012;489:57.

Thorvaldsdóttir, H., Robinson, J.T. and Mesirov, J.P. Integrative Genomics Viewer (IGV): high-performance genomics data visualization and exploration. Briefings in bioinformatics 2013;14(2):178-192.

Yang, S., et al. An Integrated Approach for RNA-seq Data Normalization. Cancer informatics 2016;15:129-141.

Yoshihara, K., et al. Inferring tumour purity and stromal and immune cell admixture from expression data. Nature communications 2013;4:2612.

Zheng, X., et al. MethylPurify: tumor purity deconvolution and differential methylation detection from single tumor DNA methylomes. Genome biology 2014;15(8):419. 
bioRxiv preprint doi: https://doi.org/10.1101/491209; this version posted April 23, 2020. The copyright holder for this preprint (which was not certified by peer review) is the author/funder, who has granted bioRxiv a license to display the preprint in perpetuity. It is made available under aCC-BY 4.0 International license.
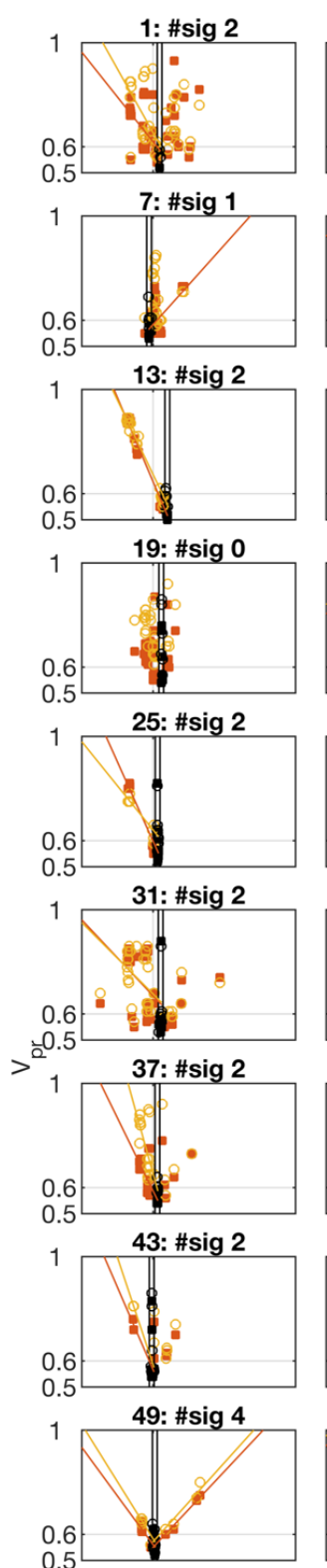

55: \#sig 3
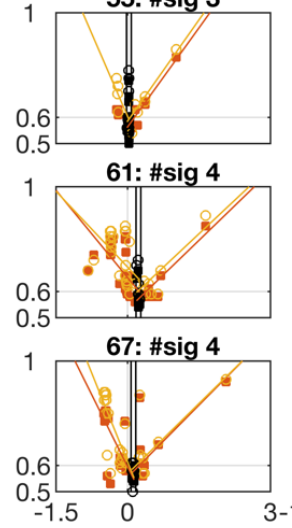

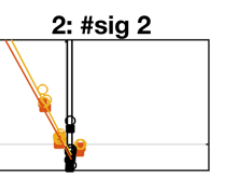

8: \#sig 2

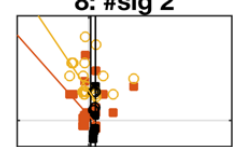

14: \#sig 4
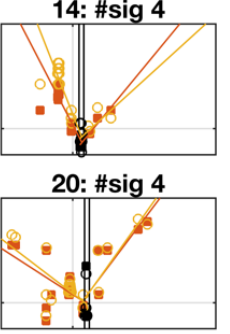

26: \#sig 0

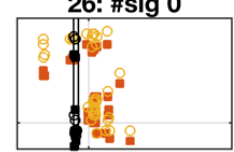

32: \#sig 0

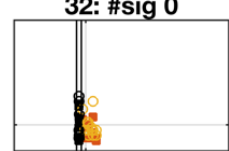

38: \#sig 2

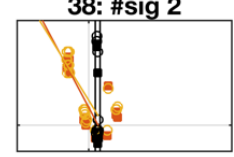

44: \#sig 0

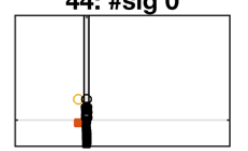

50: \#sig 4

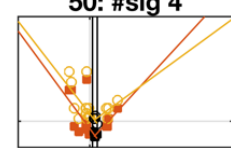

56: \#sig 2

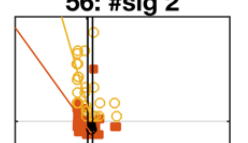

62: \#sig 4

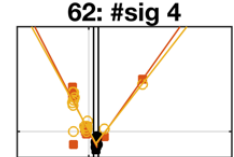

68: \#sig 4

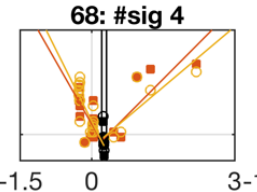

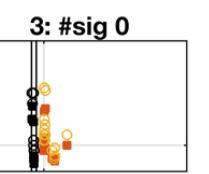

9: \#sig 0

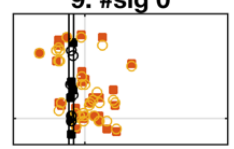

15: \#sig 3
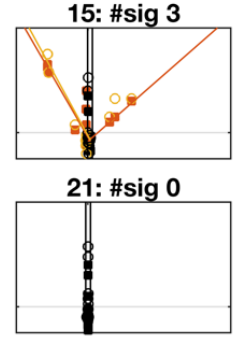

27: \#sig 2

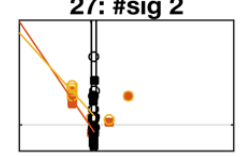

33: \#sig 4

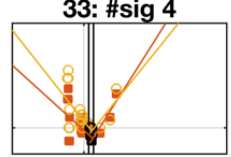

39: \#sig 2

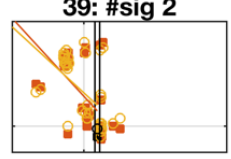

45: \#sig 4

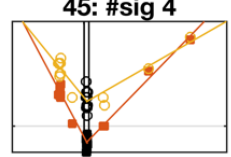

51: \#sig 3

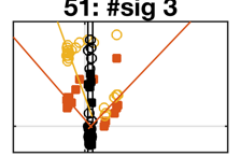

57: \#sig 2

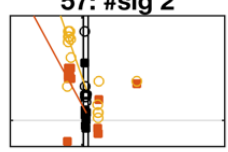

63: \#sig 2
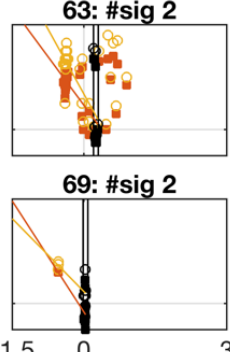

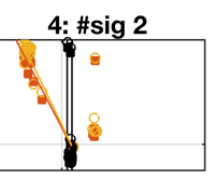

10: \#sig 0

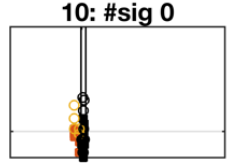

16: \#sig 4
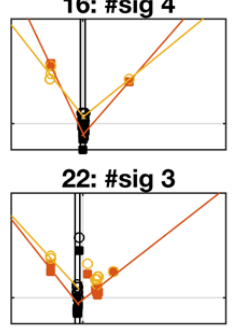

28: \#sig 4

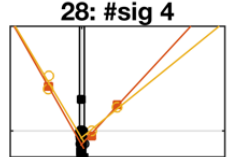

34: \#sig 4

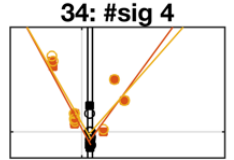

40: \#sig 4

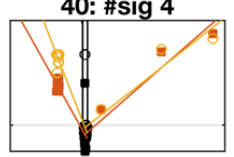

46: \#sig 4

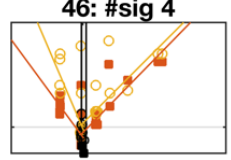

52: \#sig 4

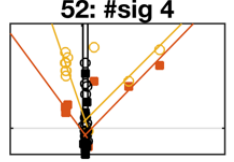

58: \#sig 0

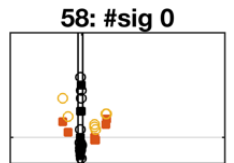

64: \#sig 3

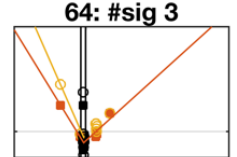

70: \#sig 2

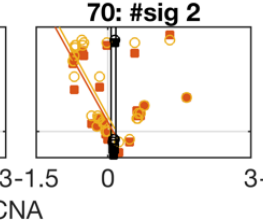

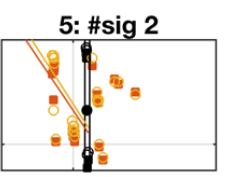

11: \#sig 4

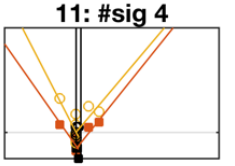

17: \#sig 4

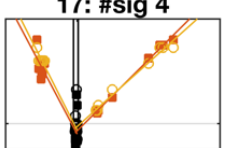

23: \#sig 2

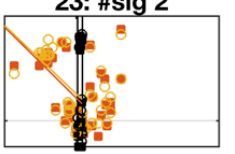

29: \#sig 4

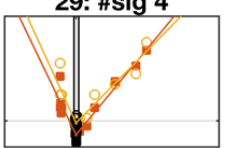

35: \#sig 4

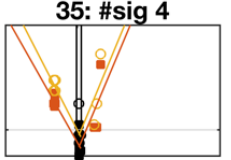

41: \#sig 4

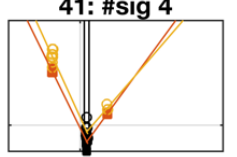

47: \#sig 4

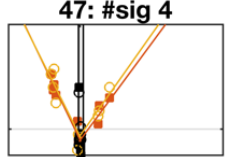

53: \#sig 4

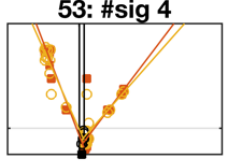

59: \#sig 4

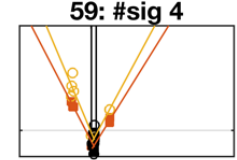

65: \#sig 1

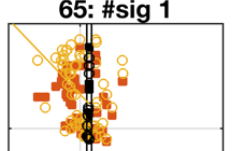

71: \#sig 4

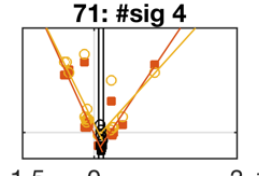

3-1.

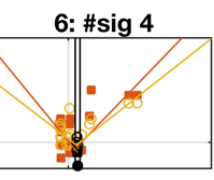

12: \#sig 2

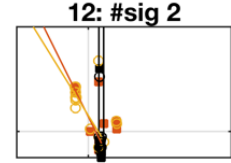

18: \#sig 4

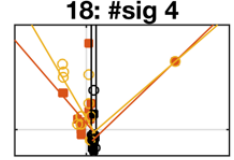

24: \#sig 3

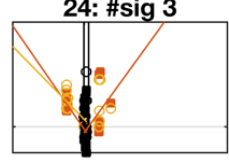

30: \#sig 0

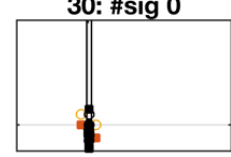

36: \#sig 2
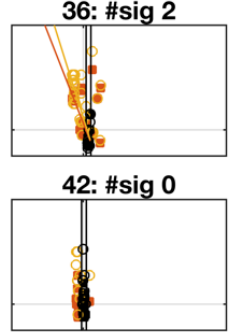

48: \#sig 4

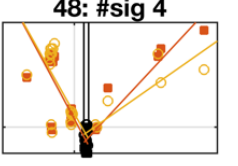

54: \#sig 4

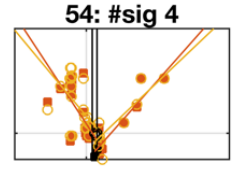

60: \#sig 4

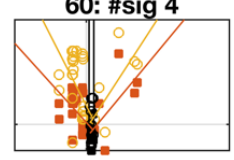

66: \#sig 3
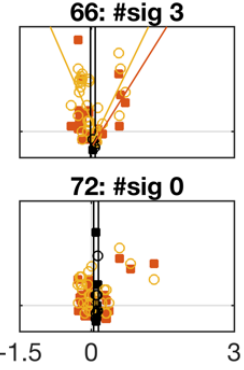

Supplementary Fig. 1. Illustration of the correlations between VPR and CNA. Orange squares VPR TEX, yellow circles VPR tR. Lines, leastsquares fitted trends for significant correlations (orange correlation with $V_{P R}, T E x$, yellow correlation with $V_{P R, T R}$ ). Black, VPR for CNAMIN \pm 0.05 . Title format Number of the dataset: \#sig number of significant correlations in the dataset. 
bioRxiv preprint doi: https://doi.org/10.1101/491209; this version posted April 23, 2020. The copyright holder for this preprint (which was not certified by peer review) is the author/funder, who has granted bioRxiv a license to display the preprint in perpetuity. It is made available under aCC-BY 4.0 International license.

Supplementary Table 1. Datasets, signals and purity estimates.

\begin{tabular}{|c|c|}
\hline \# & TCGA BRCA datasets \\
\hline 1 & $\begin{array}{l}\text { 001_Nex_BRCA_TCGA-BH-A1FC-11A_413b80f6-f6cf-4992-804a-f045e38cbe6f } \\
\text { 001_Ntr_BRCA_TCGA-BH-A1FC-11A_086db136-f3f2-42fa-aca1-63847de6ccb9 } \\
\text { 001_Tex_BRCA_TCGA-BH-A1FC-01A_1a2187a6-aea8-4096-8c3f-208a8467cd5a } \\
001 \text { Ttr_BRCA_TCGA-BH-A1FC-01A_b5e2f568-e6fc-4192-a3ab-da956e5bfa4c }\end{array}$ \\
\hline 2 & $\begin{array}{l}\text { 002_Nex_BRCA_TCGA-BH-A0B5-11A_c724807c-d80d-4582-8238-8339397b6aec } \\
002 \text { _Ntr_BRCA_TCGA-BH-A0B5-11A_f478930d-216a-40ec-b434-bfc3a7b2f62b } \\
002 \text {-Tex_BRCA_TCGA-BH-A0B5-01A_803de3d6-895f-4ad1-a86c-6f72d6ea8430 } \\
002 \text { Ttr_BRCA TCGA-BH-A0B5-01A_37175dfe-e34e-4f97-88b1-c0ba4bd5d093 }\end{array}$ \\
\hline
\end{tabular}

3003 Nex BRCA TCGA-BH-A0BJ-11A a9988fbb-090a-4363-bf73-7505e1710623 003_Ntr_BRCA_TCGA-BH-A0BJ-11A_2ced85bc-852a-4056-ad11-2e88ec6d2d82 003 Tex BRCA TCGA-BH-A0BJ-01A 58ec1111-c932-49ea-9327-1c64dfc2afa6 003 Ttr BRCA TCGA-BH-A0BJ-01A 73442f2d-3453-42ee-b57a-86871e2e2fd 9

\begin{tabular}{|c|c|c|c|c|}
\hline EST & ABS & LUMP & IHC & $\mathrm{CPE}$ \\
\hline 0.7615 & 0.49 & 0.6693 & 0.9 & 0.6517 \\
\hline 0.7539 & 0.5 & 0.8944 & 0.575 & 0.6566 \\
\hline 0.7386 & 0.37 & 0.8453 & 0.7 & 0.7458 \\
\hline 0.9534 & $\mathrm{NaN}$ & $\mathrm{NaN}$ & 0.8 & 0.7799 \\
\hline 0.909 & 0.83 & 0.9772 & 0.85 & 0.9184 \\
\hline
\end{tabular}

5005 Nex BRCA TCGA-A7-A13E-11A bd7e6f8f-7213-4ded-a8ca-3c73c7b8d918 005 Ntr_BRCA TCGA-A7-A13E-11A 99c08ce4-6526-4982-9bc7-b9c07972bcdb 005_Tex_BRCA_TCGA-A7-A13E-01A_28b8b84b-ca69-4c6a-860c-989777b18d32 005 Ttr_BRCA TCGA-A7-A13E-01A 148d5aec-6026-46b5-b40c-38a1198175ab

6006 Nex BRCA TCGA-BH-A208-11A 645b786f-1942-4cce-973b-4a75956265f5 006 Ntr BRCA TCGA-BH-A208-11A a add96f4-f194-4c8d-8757-9e8b35465a9f 006_Tex_BRCA_TCGA-BH-A208-01A_5bdbd7db-ced4-4446-9069-c44c9c1 f0ae0 006 Ttr BRCA TCGA-BH-A208-01A 794bcf95-8e66-4f91-a49c-ab10defe73c5

7007 Nex BRCA TCGA-BH-A1FU-11A db7e821b-a2b6-40e1-9fbc-c72231b703a4 007_Ntr_BRCA_TCGA-BH-A1FU-11A_c051b92b-8e11-4623-b11b-3a0d52710663 007 Tex BRCA TCGA-BH-A1FU-01A cb37bb7f-8fb6-432a-a58a-f8178d5baa64 $007^{-}$Ttr BRCA TCGA-BH-A1FU-01A 7 -

8008 Nex BRCA TCGA-BH-A0AY-11A 2ecc0325-3973-48b3-b53b-bb52aea5a9bc 008_Ntr_BRCA_TCGA-BH-A0AY-11A_-1b2877ac-94a0-464c-b58b-9ce2f16aff37 008 Tex BRCA TCGA-BH-A0AY-01A 357ccb95-03e5-49f6-ab18-38d4c8d4d820 008_Ttr_BRCA_TCGA-BH-A0AY-01A_a19a60e7-e5ca-4f66-96fe-c9add702177d

9009 Nex BRCA TCGA-BH-A18U-11A bf3d62cb-f3a6-45d6-b9c3-416e58f1d319 009_Ntr_BRCA_TCGA-BH-A18U-11A_9d4c1d7e-dd77-41d1-b1df-144e7afb2141 009 Tex BRCA TCGA-BH-A18U-01A- a80933e5-3b07-41dc-b7f0-499d63c071a9 009 Ttr_BRCA_TCGA-BH-A18U-01A_ff89e0d9-7e6c-4b6b-a1c3-f800aaa414a1

10010 Nex BRCA TCGA-AC-A2FF-11A 714e11fb-be71-4bbd-9327-457883a07ef0 $01{ }^{-}$Ntr BRCA TCGA-AC-A2FF-11A $\overline{4}$ d 32c4fa-959e-41cf-b837-104290bab9fa 010_Tex_BRCA_TCGA-AC-A2FF-01A_5c6fe1fc-839c-422a-89e7-4a54dcfad6c2 010 Ttr_BRCA TCGA-AC-A2FF-01A 37bf962c-b180-4cc2-8e0b-fde78b4f99f4

11011 Nex BRCA TCGA-BH-A0BQ-11A a5bdd116-8c1b-4787-be01-4c0f96709cc5 $011^{-}$Ntr BRCA TCGA-BH-A0BQ-11A $\overline{4}$ - 17 d22-fbed-418b-97fc-7104e1deeac 1 011_Tex_BRCA_TCGA-BH-A0BQ-01A_27138381-1865-4a6a-bd70-58725c92cb49 011 Ttr BRCA TCGA-BH-A0BQ-01A 8879454d-b803-40b6-b3d7-fbc295de9df6

12012 Nex BRCA TCGA-BH-A0BA-11A 9dbc7f19-30bd-48fd-8d5a-ca67dc26c5b1 012 Ntr BRCA TCGA-BH-A0BA-11A $2 \mathrm{cc} 17895-0 \mathrm{a} 6 \mathrm{e}-4703-8164-7034 \mathrm{f5c} 2 \mathrm{e} 1 \mathrm{a} 8$ 012 Tex BRCA TCGA-BH-A0BA-01A b4c0df66-54c1-4bbf-9a3c-d2fd28d5bb4b $012^{-}$Ttr BRCA TCGA-BH-A0BA-01A a $999701 \mathrm{c}-6 \mathrm{~b} 4 \mathrm{~b}-48 \mathrm{ed}-\mathrm{af83}-94804 \mathrm{fb} 098 \mathrm{a} 8$

13013 Nex BRCA TCGA-BH-A0B8-11A ef67ace2-01d6-4e8b-92c7-7c4e1e5ca327 013 Ntr BRCA TCGA-BH-A0B8-11A 3a833d6d-75c7-4381-8cef-699c633b64e6 013_Tex_BRCA_TCGA-BH-A0B8-01A_54972439-f9da-497d-a605-24e9670021ad 013 Ttr_BRCA TCGA-BH-A0B8-01A 9c7776d0-33df-4bd7-a720-807c650fdbc5

14014 Nex BRCA TCGA-BH-A0AU-11A 15483d36-ad24-4771-a991-8a8435effc6a 014 Ntr BRCA TCGA-BH-A0AU-11A 7 f667d91-04aa-48e8-b675-9d99b64b2058 014_Tex_BRCA_TCGA-BH-A0AU-01A_e7a641f3-cc31-4319-a04b-75c42e991711 014 Ttr BRCA TCGA-BH-A0AU-01A 23e09239-bfc3-4c2e-b690-db940d5292f7

15015 Nex BRCA TCGA-BH-A18S-11A 9e6d6a2d-ce9e-4d44-9603-f843ffa06c63 015 Ntr BRCA TCGA-BH-A18S-11A b54a0f88-21be-4c6d-a27a-1c1b8959652c 015_Tex_BRCA_TCGA-BH-A18S-01A_d4746397-9268-460a-954b-e5b5921138f9 $015^{-}$Ttr BRCA TCGA-BH-A18S-01A e 0a3ea3a-ffce-4e30-9f42-cb047a7644a1

$\begin{array}{lllll}0.5951 & 0.31 & 0.6877 & 0.6 & 0.5642\end{array}$

0.6835 
bioRxiv preprint doi: https://doi.org/10.1101/491209; this version posted April 23, 2020. The copyright holder for this preprint (which was not certified by peer review) is the author/funder, who has granted bioRxiv a license to display the preprint in perpetuity. It is made available under aCC-BY 4.0 International license.

16 016_Nex_BRCA_TCGA-BH-A0HK-11A_d256dce0-d74b-4f8f-bf47-40b1b953fc7f 016 Ntr BRCA TCGA-BH-A0HK-11A $438650 \mathrm{e} 8-0 \mathrm{ee} 2-4 \mathrm{c} 74-8432-88 \mathrm{~b} 5 \mathrm{c} 8006187$ 016_Tex_BRCA_TCGA-BH-A0HK-01A_944b4c29-bf72-4eec-b277-badc237730de 016_Ttr_BRCA_TCGA-BH-A0HK-01A_fe04f368-0a73-4f97-9d6b-2986f9b2b052

17 017_Nex_BRCA_TCGA-A7-A0D9-11A_dda70534-0d4d-4c30-9c6a-fb3c39396fb0 $017^{-}$Ntr_BRCA TCGA-A7-A0D9-11A 17 cf6364-e228-4ee9-bffa-d1ad75f4152b 017_Tex_BRCA_TCGA-A7-A0D9-01A_821d7a33-77fb-496e-be9c-0552b12cbbee 017_Ttr_BRCA_TCGA-A7-A0D9-01A_c0ecd314-9d99-48ec-83f1-5a0c1ed656aa

18 018_Nex_BRCA_TCGA-BH-A0BV-11A_56dfc492-2b1f-4494-9ba9-14a70601ae21 018 Ntr BRCA TCGA-BH-A0BV-11A 20459115-d7be-4d04-896f-c5ff6923ec4c 018 Tex BRCA TCGA-BH-A0BV-01A beb9e4cf-1f76-4a26-acee-e88d0936e60b 018_Ttr_BRCA_TCGA-BH-A0BV-01A_d037d3c2-e316-473d-9970-d4fb43615d95

19 019_Nex_BRCA_TCGA-E2-A1LH-11A_61558dd3-8f6c-4f70-8717-7676580fa5a7 019 Ntr BRCA TCGA-E2-A1LH-11A c7e02b93-465f-47da-81d7-ec9a8cb1e52b 019 Tex BRCA TCGA-E2-A1LH-01A f54770bb-5dd0-48cf-ac5a-3f023a6aef95 019_Ttr_BRCA_TCGA-E2-A1LH-01A_169c390c-a211-4db0-a983-9bf5d6eee16e

20 020_Nex_BRCA_TCGA-BH-A0DD-11A_e9fe9b97-f7c7-40dc-ae31-17bb15c9fd8b 020 Ntr BRCA TCGA-BH-A0DD-11A 5482cdd0-3698-455b-97c1-b10c69d67ae9 020 Tex BRCA TCGA-BH-A0DD-01A 99ca9706-f2bf-430b-9b23-e0947c0f8593 020_Ttr_BRCA_TCGA-BH-A0DD-01A_90cbc532-1ca8-46d6-977c-72b6d01e9c34

21 021_Nex_BRCA_TCGA-BH-A0H5-11A_adfb1a86-fbb1-4b71-9c04-f99399f20d70 021 Ntr BRCA TCGA-BH-A0H5-11A 896d76a1-bae8-495a-9e12-e82e16bd8b16 021_Tex_BRCA_TCGA-BH-A0H5-01A_6cc3c90e-c77c-4609-ada5-9b78c659dc34 021_Ttr_BRCA_TCGA-BH-A0H5-01A_778c9326-998d-4081-b148-0eede2b94e29

22 022_Nex_BRCA_TCGA-A7-A0DB-11A_91081819-79c8-4de6-bfdb-742df760c08b 022 Ntr BRCA TCGA-A7-A0DB-11A a 8ed2ec3-0285-4028-9698-710a148ce11b 022_Tex_BRCA_TCGA-A7-A0DB-01A_37a9daca-9d53-4ec4-8de2-dc2c140a5d8f 022 Ttr BRCA TCGA-A7-A0DB-01A_1f62e969-d05d-4a4d-a163-cb06e4958f71

23 023_Nex_BRCA_TCGA-BH-A1FN-11A_e1c0d95f-949c-4cec-9cf8-f91c3b90b8d9 023 Ntr_BRCA TCGA-BH-A1FN-11A d5e5f3c9-4129-4c92-87f3-6f86577a7584 023 Tex BRCA TCGA-BH-A1FN-01A 8d715491-6943-4d58-92f6-88cce7b463e2 023_Ttr_BRCA_TCGA-BH-A1FN-01A_8e7dc738-8a8f-45b2-bd82-4125a07d7373

24 024_Nex_BRCA_TCGA-BH-A0AZ-11A_9bbae9a0-9f12-48cf-9aa7-d070c6627ea5 024 Ntr BRCA TCGA-BH-A0AZ-11A 693bf8e4-b266-4b58-b812-f579179efb65 024_Tex_BRCA_TCGA-BH-A0AZ-01A_664528b7-b511-4627-8464-0702263434c5 024_Ttr_BRCA_TCGA-BH-A0AZ-01A_07f377f4-0bd1-4647-bf06-ff6ed553c44a

25 025_Nex_BRCA_TCGA-BH-A0HA-11A_c61bb1ab-688f-4d58-8388-60ae77c28840 $025^{-}$Ntr BRCA TCGA-BH-A0HA-11A 09e 07a68-a443-4c16-a0de-78cd8aea59c0 025 Tex BRCA TCGA-BH-A0HA-01A_ 2c144eba-6490-4d64-9446-085d6edc8308 025_Ttr_BRCA_TCGA-BH-A0HA-01A_6d483def-2d91-4afc-991a-4a29804a6f3a

026_Nex_BRCA_TCGA-A7-A0CE-11A_eee8d4d0-d524-47f5-b076-6ad6216de1a3 026 Ntr BRCA TCGA-A7-A0CE-11A 548cad87-ec95-47e2-890e-7c8284ea5b88 026 Tex BRCA TCGA-A7-A0CE-01A 4288da4e-7e77-434b-a092-9450b0cb7833 026_Ttr_BRCA_TCGA-A7-A0CE-01A_14201682-0c8d-49c7-a5e1-7026e1a07b69

27 027_Nex_BRCA_TCGA-BH-A0DK-11A_3f4400a1-84ab-4198-b9a1-67b2ffc5ef36 027 Ntr BRCA TCGA-BH-A0DK-11 A ae67044f-62c9-405f-bfc1-f0b8f1bc66d3 $027^{-}$Tex BRCA TCGA-BH-A0DK-01A e3e2053a-3ca2-4527-9b94-209def68dcc3 027_Ttr_BRCA_TCGA-BH-A0DK-01A_a3df35ec-a8d2-44ad-8ba6-eaba504261e0

28 028_Nex_BRCA_TCGA-BH-A0E1-11A_f6fed4ed-a853-40aa-bf7b-e627efd402d6 028 Ntr BRCA TCGA-BH-A0E1-11A 52441de4-e26b-42b3-b061-94907c049501 028 Tex BRCA TCGA-BH-A0E1-01A 3c7e6a59-08b8-4903-932a-99946a96b746 028_Ttr_BRCA_TCGA-BH-A0E1-01A_f412f8d8-9e35-41d9-b44a-131186cb4bb0

29 029_Nex_BRCA_TCGA-BH-A0DG-11A_c99b1 fb3-17e3-4472-86ee-7fda358a92c2 029 Ntr BRCA TCGA-BH-A0DG-11A bfdaf242-1e97-450d-9983-2cbb4e99305d 029 Tex BRCA TCGA-BH-A0DG-01A 721e2f71-60ae-4d63-9f05-113bce56c672 029_Ttr_BRCA_TCGA-BH-A0DG-01A_865afd6b-84a7-4dde-aa23-0b925c0b9d50

30 030_Nex_BRCA_TCGA-AC-A2FB-11A_552279ea-d7b1-496d-8170-ca30f5b62b5a 030 Ntr BRCA TCGA-AC-A2FB-11A 56cd7da0-2c47-4986-91ce-07db2bb87369 030 Tex BRCA TCGA-AC-A2FB-01A de000c35-8bf4-470a-9656-1b5da0deebe6 030_Ttr_BRCA_TCGA-AC-A2FB-01A_35aa5078-e07f-4a0f-84c1-01a0e566e $97 \mathrm{c}$
0.78

0.9163

0.925

0.8357

0.775

0.8921

0.6895

$\mathrm{NaN}$

0.725

0.6749

0.5948

0.32

0.5637

0.8

0.4633

0.8677

0.945

0.625

0.8714

0.4399

$\mathrm{NaN}$

$\mathrm{NaN}$

0.475

0.1632

0.7341

$\mathrm{NaN}$

0.85

0.6494

0.8367

0.7

0.8509

0.75

0.8313

0.6505

0.53

0.8696

0.7

0.6655

0.6418

0.74

0.9258

0.725

0.7386

0.9035

0.73

$\mathrm{NaN}$

0.835

0.8551

0.5384

0.53

0.7316

0.7

0.6837

0.8676

0.974

0.825

0.8524

0.6358 
bioRxiv preprint doi: https://doi.org/10.1101/491209; this version posted April 23, 2020. The copyright holder for this preprint (which was not certified by peer review) is the author/funder, who has granted bioRxiv a license to display the preprint in perpetuity. It is made available under aCC-BY 4.0 International license.

31031 Nex BRCA TCGA-BH-A0H7-11A abfca562-d328-40d2-83bb-e584123b0f28 031_Ntr_BRCA_TCGA-BH-A0H7-11A_d969d9b2-9d8b-4594-95d4-87e6ce1236fc 031_Tex_BRCA_TCGA-BH-A0H7-01A_e8daad78-39fc-4835-b1c4-8807653d9c9a 031_Ttr_BRCA_TCGA-BH-A0H7-01A_0d37f87a-760a-472a-acba-bbc255422fbe

32 032 Nex BRCA TCGA-BH-A1EU-11A 38e87966-9605-4454-a4d1-28f96b7689f7 032 Ntr BRCA TCGA-BH-A1EU-11A 3b00c121-17f2-461e-8873-08d15c9ec9f4 032 Tex BRCA TCGA-BH-A1EU-01A 4bccbb0f-2641-44df-b89a-42f020b4c08f 032 Ttr BRCA TCGA-BH-A1EU-01A 86e3dba1-48fb-44cc-b046-8bc35963ce99

33033 Nex BRCA TCGA-BH-A0DP-11A 27543260-52ac-444b-8214-e62dca2cc8fe 033_Ntr_BRCA_TCGA-BH-A0DP-11A_30f4e5d8-a13d-4ef2-88e 0-a01e 07c2e142 033_Tex_BRCA_TCGA-BH-A0DP-01A_0326975a-2e56-404a-8776-92c5c5678853 033 Ttr_BRCA TCGA-BH-A0DP-01A 7ff8a7a0-5235-4de0-bb9f-b811230b5bda

34034 Nex_BRCA TCGA-BH-A18N-11A 6c8aae77-5f43-41ec-a139-81f3ba02f6ea 034_Ntr_BRCA_TCGA-BH-A18N-11A_0738f1b2-aa50-4921-82e1-d3614b40f98d 034_Tex_BRCA_TCGA-BH-A18N-01A_b6f89799-9070-4fbc-b10c-53cbe515eccc 034 Ttr_BRCA TCGA-BH-A18N-01A_4b7b8eb8-d939-411c-be5e-cf41a5521963

35035 Nex BRCA TCGA-BH-A0BC-11A 6359db46-f8dd-4dc8-a3a9-8725d8f6958a 035_Ntr_BRCA_TCGA-BH-A0BC-11A_2cb50d4a-d6df-4b64-acfb-7a7db5ddd1de 035_Tex_BRCA_TCGA-BH-A0BC-01A_73b9208d-336c-4990-a27d-0164a77dd165 035 Ttr BRCA TCGA-BH-A0BC-01A 92e26b53-f540-428a-a3c5-848a36b31171

36036 Nex BRCA TCGA-BH-A0BZ-11A 2b4e3d99-07cd-4b06-ad97-82a19ac0eb5d 036_Ntr_BRCA_TCGA-BH-A0BZ-11A_3aa16a4b-4e35-4530-84fb-0cb204290b08 036_Tex_BRCA_TCGA-BH-A0BZ-01A_74414845-839f-4885-b13d-3f2e17781f84 036 Ttr BRCA_TCGA-BH-A0BZ-01A efefcc2f-72e9-4634-b943-d26083e1a312

37037 Nex BRCA TCGA-BH-A0DL-11A 2d495f9c-4ffa-4169-b583-6786612e9606 037 Ntr BRCA TCGA-BH-A0DL-11A bd8b100a-8391-4046-847f-c3fdd3830eeb $037^{-}$Tex BRCA TCGA-BH-A0DL-01A dfd355e4-478a-47cb-9aab-8ce22b6f936c 037 Ttr BRCA TCGA-BH-A0DL-01A 11d77ef2-b3f9-4af9-8490-71f9a8c599e0

38038 Nex BRCA TCGA-BH-A0BT-11A 32430467-5215-4738-86a3-5bbe11fbba86 038 Ntr BRCA TCGA-BH-A0BT-11A cbef4196-5f3b-40d9-b26f-5b2bb82fbe9b 038_Tex_BRCA_TCGA-BH-A0BT-01A_e 78b9962-7bc2-4238-806a-5933ac07de99 038 Ttr BRCA TCGA-BH-A0BT-01A aae75165-efa0-46b3-8a8d-82dc7d82aecd

39039 Nex BRCA TCGA-BH-A18Q-11A b58d4f69-a4ea-489b-9d25-e5cfdc465adb 039 Ntr BRCA TCGA-BH-A18Q-11A 76575097-374b-4fb2-8054-2a31b4204165 039_Tex_BRCA_TCGA-BH-A18Q-01A_1f2c90ef-a05d-494c-9232-e705691f46b9 039 Ttr BRCA_TCGA-BH-A18Q-01A f0173e28-7fe4-411f-a187-57fd94a7935a

40 040 Nex BRCA TCGA-E2-A1LB-11A e2d7a695-b0bf-4432-8f98-1843bb49efba 040 Ntr BRCA TCGA-E2-A1LB-11A 6eb518ab-f174-45ae-8d65-74086ecb1125 040_Tex_BRCA_TCGA-E2-A1LB-01A_3ddbc444-ee1b-43be-bab5-b0f67d5eb339 040 Ttr BRCA TCGA-E2-A1LB-01A d7d566a0-b6d0-4a4f-8211-9309b27b0ade

41041 Nex BRCA TCGA-BH-A0DH-11A a7a7e0f6-100f-4145-9599-693e6c14e903 041 Ntr BRCA TCGA-BH-A0DH-11A 5a0374e5-cee9-4952-9df0-4ff125196478 041_Tex_BRCA_TCGA-BH-A0DH-01A_eb680f8c-4ba1-45ef-8b94-e58b68922f2f 041 Ttr BRCA TCGA-BH-A0DH-01A 71a3c27c-0982-4da6-b260-cf16a4868a19

42 042 Nex BRCA TCGA-BH-A0B7-11A d9aca915-ea30-4939-af59-edaef8872396 042 Ntr BRCA TCGA-BH-A0B7-11A 8db8b247-05b8-46ca-8791-ecf846da2c7f 042_Tex_BRCA_TCGA-BH-A0B7-01A_e3b9eb8a-93f3-4668-a54b-fa8b15be5667 042 Ttr_BRCA_TCGA-BH-A0B7-01A_0fdae4ee-ca68-4ba4-ba58-76058409b02f

43043 Nex BRCA TCGA-E2-A15I-11A 36024763-f828-4496-8fdc-46d5c3de569b 043 Ntr BRCA TCGA-E2-A15I-11A ffa9ace8-9253-4775-9ad2-2a8a50c0f9c9 043_Tex_BRCA_TCGA-E2-A15I-01A_8c627466-eb99-4a7e-87e6-314ae8ed32a1 043 Ttr BRCA TCGA-E2-A15I-01A 3a4e3785-fb2e-4ffc-9644-91c78a9a9ebe

44044 Nex_BRCA TCGA-BH-A0DV-11A 79a92eab-c87c-4209-819e-193d653c0df6 044 Ntr BRCA TCGA-BH-A0DV-11A e 87e7e3e-9059-47cf-9f45-8959250b037f 044_Tex_BRCA_TCGA-BH-A0DV-01A_105290eb-b626-4318-9b8a-42f477e2cec6 044 Ttr BRCA TCGA-BH-A0DV-01A 7 bad3f4c-6065-4245-8119-c25596f38829

45045 Nex BRCA TCGA-BH-A0DZ-11A aebf04d4-4a1b-4a50-b5f1-0f9e2c273121 045 Ntr BRCA TCGA-BH-A0DZ-11A 80b4d43d-9e7d-4ab8-b05a-0eb51faa9d12 045_Tex_BRCA_TCGA-BH-A0DZ-01A_4e7d62f5-4be9-4b9c-9b7c-aec4567dded2 045 Ttr BRCA TCGA-BH-A0DZ-01A 8b1982a0-315c-47e1-8de0-a1e5ec51dd74

46046 Nex BRCA TCGA-BH-A18R-11A b32b2067-a79e-42c5-ae78-135c845253fe 046 Ntr BRCA TCGA-BH-A18R-11A f82099ae-9d74-44d8-ba5b-cd10eeb09807
0.7939

$0.9561 \quad 0.725$

0.7534

0.5387

$0.33 \quad 0.6869 \quad 0.65$

0.4299

0.7037

$\begin{array}{lll}0.42 & 0.8565 & 0.7\end{array}$

0.655

0.868

$0.76 \quad \mathrm{NaN}$

0.9

0.832

0.6221

0.6

0.8221

0.8

0.7727

0.5788

0.37

0.7363

0.6

0.5138

0.6944

0.53

$\mathrm{NaN}$

0.65

0.6655

0.8096

0.67

0.9054

0.75

0.7751

0.81

$0.73 \quad \mathrm{NaN}$

0.9

0.836

0.84

0.68

0.8192

0.9

0.815

0.83

0.76

0.8955

0.85

0.8597

$\begin{array}{lllll}0.6207 & 0.2 & \mathrm{NaN} & 0.575 & 0.4954\end{array}$

$\begin{array}{lllll}0.7689 & 0.62 & 0.768 & 0.8 & 0.7565\end{array}$

$\begin{array}{lllll}0.6021 & 0.31 & 0.7631 & 0.7 & 0.4856\end{array}$

$\begin{array}{lllll}0.6572 & 0.65 & \mathrm{NaN} & 0.885 & 0.7792\end{array}$

$\begin{array}{lllll}0.8685 & 0.53 & \mathrm{NaN} & 0.85 & 0.7466\end{array}$ 
bioRxiv preprint doi: https://doi.org/10.1101/491209; this version posted April 23, 2020. The copyright holder for this preprint (which was not certified by peer review) is the author/funder, who has granted bioRxiv a license to display the preprint in perpetuity. It is made available under aCC-BY 4.0 International license.

046 Tex BRCA TCGA-BH-A18R-01A c518bc34-50dc-4265-824f-a954e4d19f0b 046_Ttr_BRCA_TCGA-BH-A18R-01A_fc65ff2e-9808-4c1e-a16b-8285fd0d27df

47 047_Nex_BRCA_TCGA-E2-A15K-11A_6299f114-932a-42c0-8cab-bebb12c996fc $047^{-}$Ntr BRCA TCGA-E2-A15K-11A c2ab9488-d9a4-479c-b9e2-6f9b0cdbaacb 047 Tex BRCA TCGA-E2-A15K-01A 80019ec7-b0d8-4573-b5d6-a5d9f2745ab2 047_Ttr_BRCA_TCGA-E2-A15K-01A_7e3a600b-edd8-428e-b88d-af4c63dcaad9

48 048_Nex_BRCA_TCGA-BH-A1EN-11A_a6259119-3d8a-4749-a517-c675efbc8215 048 Ntr BRCA TCGA-BH-A1EN-11A 488f1b69-c2a3-429b-a972-31edfd615a67 048 Tex BRCA TCGA-BH-A1EN-01A 72e4cb26-911c-4804-9e4f-ed5b51024cd1 048_Ttr_BRCA_TCGA-BH-A1EN-01A_96360e75-26b6-4647-b974-9e31ae6de00c

049 Nex BRCA TCGA-BH-A0H9-11A d8b452e5-010a-4fec-80a4-770a5a492090 049_Ntr_BRCA_TCGA-BH-A0H9-11A_1337ceba-db77-4b31-ac20-1c6a8bb5f546 049 Tex BRCA TCGA-BH-A0H9-01A ac4899fe-f56d-4b98-9a54-73ffd0c90652 049_Ttr_BRCA_TCGA-BH-A0H9-01A_ä97d281f-235f-481b-b26b-169b96e0e65f

50050 Nex BRCA TCGA-E2-A153-11A 1c3f2e11-952a-4e47-a8b9-25f4fe4bf205 050_Ntr_BRCA_TCGA-E2-A153-11A_bobd0ab51c-c114-40e3-a6a1-4b8f576a41d3 050 Tex BRCA TCGA-E2-A153-01A 85258fb2-26ab-4a66-b8a5-5a58bf9275e0 050_Ttr_BRCA_TCGA-E2-A153-01A_091a54cd-e3b3-4af2-828f-a80e64504f5e

51051 Nex BRCA TCGA-BH-A0BW-11A ab130f7f-4070-436e-ac7b-c1b7aecb9dc6 051 Ntr BRCA TCGA-BH-A0BW-11A 2581c95b-1b57-4407-bbc4-a65c89bfd136 051 Tex BRCA TCGA-BH-A0BW-01A 7661179f-df6c-4a57-adca-224b62d98348 051_Ttr_BRCA_TCGA-BH-A0BW-01A_a15d171e-ac82-4712-94a9-b4799e7b2915

52052 Nex BRCA TCGA-BH-A18J-11A 3aa5b173-17b2-425f-b5c7-395614d6bfa2 052_Ntr_BRCA_TCGA-BH-A18J-11A_307eb339-a781-45cc-9597-da0be7e5438a 052 Tex BRCA TCGA-BH-A18J-01A f639a485-8ebb-4dcc-9f2e-a8d7ad05564f 052_Ttr_BRCA_TCGA-BH-A18J-01A_0e985713-0492-4191-918b-fef6c23389b1

53053 Nex BRCA TCGA-BH-A204-11A 98e5c1d8-5c14-4416-8437-31d0098dd341 053_Ntr_BRCA_TCGA-BH-A204-11A_2afdc0cf-2723-42dd-89f7-fa03c6ba218c 053 Tex BRCA TCGA-BH-A204-01A 970600ce-9486-4641-8555-533132f7a414 053_Ttr_BRCA_TCGA-BH-A204-01A_893fcf87-8baa-424e-8866-bcf8cfa26cf9

54054 Nex BRCA TCGA-BH-A18K-11A 502ee86f-829e-4b6e-a8f1-be082c445310 054_Ntr_BRCA_TCGA-BH-A18K-11A_375bcdd8-8628-4047-948a-fa98bfa3dba5 054 Tex BRCA TCGA-BH-A18K-01A 7e8c2ea7-04ce-47c5-b848-229f96563015 054_Ttr_BRCA_TCGA-BH-A18K-01A_12473f59-359c-4306-ade3-2156e458cd05

55055 Nex BRCA TCGA-BH-A0C3-11A 9fedd2f4-d2c8-4d24-987b-69edf55e15f1 055_Ntr_BRCA_TCGA-BH-A0C3-11A_a49fa48d-efc4-4b99-a42e-7019236af6c8 055 Tex BRCA TCGA-BH-A0C3-01A 866d9cb0-a299-46c1-a787-2e73fd758fbe 055_Ttr_BRCA_TCGA-BH-A0C3-01A_ 164f86df-dec9-44ef-b1c7-2ee5d33617be

56056 Nex BRCA TCGA-BH-A0C0-11A 9778035c-19ff-4a89-bba2-fa83e51d9add 056_Ntr_BRCA_TCGA-BH-A0C0-11A_52a72824-0b41-4b8e-86f0-41ee5e00c989 056 Tex BRCA TCGA-BH-A0C0-01A 568a2363-b7e5-48f6-9242-328950eebf39 056_Ttr_BRCA_TCGA-BH-A0C0-01A_f5fe9655-f5b4-413b-882c-43b872e4ec 23

57057 Nex BRCA TCGA-BH-A0E0-11A 59fa57c8-7435-4499-bd09-bf969596c18d 057 Ntr BRCA TCGA-BH-A0E0-11A a 3e5f7bd-3ab0-4ea6-9de1-742de1a2ed 78 057 Tex BRCA TCGA-BH-A0E0-01A 72436fcd-21fd-46bd-bd36-7f514edb51de 057_Ttr_BRCA_TCGA-BH-A0E0-01A_b $98 \mathrm{~d} 2 \mathrm{a} 16-974 \mathrm{c}-4728-9648-81 \mathrm{dc} 4314 \mathrm{f} 225$

58058 Nex BRCA TCGA-BH-A18M-11A 8e0cb775-9fc9-4001-973c-ef6cec2a38b6 058_Ntr_BRCA_TCGA-BH-A18M-11A_3e02aa37-30ee-4663-bba1-280e5127f302 058 Tex BRCA TCGA-BH-A18M-01A 69ccc418-264c-4e8e-a034-39607c07fa59 058_Ttr_BRCA_TCGA-BH-A18M-01A_aea8fff8-dbc9-4a1b-9a3a-ca1882432c57

59 059_Nex_BRCA_TCGA-E2-A1BC-11A_45b8b995-c477-4358-8050-6d41c267b467 059 Ntr BRCA TCGA-E2-A1BC-11A 205007dd-4bc1-4e1f-9fdb-115b0e7c9836 059_Tex_BRCA_TCGA-E2-A1BC-01A_f969cce4-0fcd-47bb-91f1-37ba0f314994 059 Ttr BRCA TCGA-E2-A1BC-01A ea3bd91d-520b-4198-b011-a0f578eadc3e

60060 Nex BRCA TCGA-BH-A203-11A f08939ed-a218-4688-b419-a91333d0267b 060 Ntr BRCA TCGA-BH-A203-11A 8c2d82aa-0b36-488f-8cf1-f82795b831c5 060_Tex_BRCA_TCGA-BH-A203-01A_55a9b84d-ca9f-402c-8d93-15aef2fde988 060 Ttr BRCA TCGA-BH-A203-01A 8986bdd8-2be5-41ed-9596-59ca1f95e1c0

61061 Nex BRCA TCGA-BH-A1F2-11A d6eb2d94-1234-46b3-9403-958f3b340fd0 061 Ntr BRCA TCGA-BH-A1F2-11A 210014fa-f161-4799-a1c0-6f93d4b631f6 061_Tex_BRCA_TCGA-BH-A1F2-01A_91aeda5a-ed5a-4175-b19e-408219b980fc 061 Ttr BRCA TCGA-BH-A1F2-01A ceb5a503-e107-4721-9283-714406cdd914
0.7

0.724

0.9

$\mathrm{NaN}$

0.8518

0.7

0.9612

0.85

0.8179

0.8872

0.35

0.7934

0.6589

0.61

$\mathrm{NaN}$

0.6

0.5474

0.6784

$0.54 \mathrm{NaN}$

0.6

0.6749

0.8066

$0.51 \quad \mathrm{NaN}$

0.7243

0.8763

0.8058

0.58

$\mathrm{NaN}$

0.8

0.7468

0.6289 
bioRxiv preprint doi: https://doi.org/10.1101/491209; this version posted April 23, 2020. The copyright holder for this preprint (which was not certified by peer review) is the author/funder, who has granted bioRxiv a license to display the preprint in perpetuity. It is made available under aCC-BY 4.0 International license.

62 062_Nex_BRCA_TCGA-BH-A1EO-11A_90308930-e3c5-47bb-bcee-58eaae7d3dfa 062 Ntr BRCA TCGA-BH-A1EO-11A a 426d9c2-86b1-4db1-b49c-eceaa01273a9 062 Tex BRCA TCGA-BH-A1EO-01A 7787e3e2-f604-4b4d-a3bc-60c795d4177b 062_Ttr_BRCA_TCGA-BH-A1EO-01A_e31bd4a4-ecda-49b4-83b0-7f1496c2f9ae

63 063_Nex_BRCA_TCGA-BH-A18V-11A_353e7fa1-08c5-400a-b352-b5325e40d66c 063 Ntr BRCA TCGA-BH-A18V-11A e3c5cba8-e3ba-4e0b-929b-280708e0a855 063 Tex BRCA TCGA-BH-A18V-01A abcf2a8e-6f4c-4668-9ef9-41d95d16e8e6 063_Ttr_BRCA_TCGA-BH-A18V-01A__286394db-7d5e-4de2-b386-581352164350

64 064_Nex_BRCA_TCGA-BH-A0DT-11A_6dde640a-1d79-4e7d-9491-c500b8183d9a 064 Ntr BRCA TCGA-BH-A0DT-11A 71 aa4cd6-75ea-4e10-b16c-ea9adbf31a98 064 Tex BRCA TCGA-BH-A0DT-01A 9d93c6fb-336a-4cb4-9f33-8557456753b1 064_Ttr_BRCA_TCGA-BH-A0DT-01A_61ad7408-dacd-4913-a479-c456e8b03191

065_Nex_BRCA_TCGA-GI-A2C9-11A_454dbdba-da53-4e99-9670-dff1e5bbb77c 065_Ntr_BRCA_TCGA-GI-A2C9-11A d d8aa0349-d74e-4891-8398-6476eb1935f0 065 Tex BRCA TCGA-GI-A2C9-01A 2f2b0909-488b-4fa3-8251-2ef6e7d5869e 065_Ttr_BRCA_TCGA-GI-A2C9-01A_01ea694e-989b-4a35-9397-5e508656d1d8

066_Nex_BRCA_TCGA-BH-A209-11A_c580c610-832d-45be-9963-06bb918ede73 066_Ntr_BRCA_TCGA-BH-A209-11A_b8b48554-ca2f-466d-85b0-9d473cca8ca7 066 Tex BRCA TCGA-BH-A209-01A 7b85ca36-2fe9-4156-99eb-f79463dbc572 066_Ttr_BRCA_TCGA-BH-A209-01A_b2cf947a-5ed1-4e24-8752-bf2a6eca895a

67067 Nex BRCA TCGA-BH-A1EV-11A f4d30842-7873-46d3-8f25-ae7d05909175 067_Ntr_BRCA_TCGA-BH-A1EV-11A_73e296db-9eec-4060-97c9-80a98dbb9fb6 067 Tex BRCA TCGA-BH-A1EV-01A fb502696-cb13-487a-a70a-6ceefcf20ca0 067_Ttr_BRCA_TCGA-BH-A1EV-01A_93ab3adf-7ab9-455e-9007-9f51443352fe

68068 Nex BRCA TCGA-GI-A2C8-11A 836e4482-11c7-4422-a2e5-cac9b846ea71 068_Ntr_BRCA_TCGA-GI-A2C8-11A_580d9a3d-e198-4e7b-aa1f-419d868bb0b5 068 Tex BRCA TCGA-GI-A2C8-01A 146c0ba4-6761-446c-be7c-7e56c0ffa37b 068_Ttr_BRCA_TCGA-GI-A2C8-01A_c0ee6e25-02b9-4b2f-9f23-fd61 eedf9945

69069 Nex BRCA TCGA-BH-A0BM-11A 92faafbd-6a76-4116-80fc-a15767aa81d0 069_Ntr_BRCA_TCGA-BH-A0BM-11A_ae127be2-5e4c-4b7e-9cf8-3aa9e529baaa 069 Tex BRCA TCGA-BH-A0BM-01A c513ed81-255f-43b0-b8aa-984326201745 069_Ttr_BRCA_TCGA-BH-A0BM-01A_006b2b95-7069-4cb6-bfe8-7edb80056add

70070 Nex BRCA TCGA-BH-A18L-11A 7a010ccd-f780-45f0-98da-cc738e87b6d3 070_Ntr_BRCA_TCGA-BH-A18L-11A_e ef4660c0-c177-4d46-90f9-56e3dc47b59e 070 Tex BRCA TCGA-BH-A18L-01A 0d4aca9c-c11e-4f78-a250-08d45ce4828 070_Ttr_BRCA_TCGA-BH-A18L-01A_1 af43803-7afa-4d2b-aa78-2dec84c1e702

71071 Nex BRCA TCGA-A7-A13F-11A 471d1e10-7c79-44f9-a373-bd3e510b6155 071_Ntr_BRCA_TCGA-A7-A13F-11A_4e4cd9e5-27bb-4ea7-9328-0b267373ec1c 071 Tex BRCA TCGA-A7-A13F-01A d8fad6b2-66b8-4d6f-b018-653998675921 071_Ttr_BRCA_TCGA-A7-A13F-01A_75898a6d-75e4-4dca-a7ed-c11056e0c9c4

72072 Nex BRCA TCGA-E2-A15M-11A b2138cda-519f-4691-bf1c-0f863b55d888 072_Ntr_BRCA_TCGA-E2-A15M-11A_bf873756-8ee8-49bd-b2ca-17223c7ef962 072 Tex BRCA TCGA-E2-A15M-01A 1ccf392d-7959-4bb9-8ca8-4298409f4951 072_Ttr_BRCA_TCGA-E2-A15M-01A_b2569032-6147-4a1c-973f-d5985127e9f4

\begin{tabular}{|c|c|c|c|c|}
\hline 0.5849 & 0.48 & 0.8854 & 0.9 & 0.7493 \\
\hline 0.6941 & 0.56 & $\mathrm{NaN}$ & 0.75 & $\mathrm{NaN}$ \\
\hline 0.7462 & 0.42 & $\mathrm{NaN}$ & 0.55 & 0.6659 \\
\hline 0.8062 & 0.51 & 0.8386 & 0.8 & 0.6969 \\
\hline 0.5979 & 0.24 & 0.6243 & 0.6 & 0.4645 \\
\hline 0.8435 & 0.61 & 0.9517 & 0.8 & 0.817 \\
\hline 0.601 & 0.48 & 0.7846 & 0.85 & 0.6998 \\
\hline 0.796 & 0.61 & 0.9189 & 0.84 & 0.7886 \\
\hline 0.927 & 0.81 & $\mathrm{NaN}$ & 0.8 & 0.9113 \\
\hline 0.8478 & 0.65 & 0.9236 & 0.7 & 0.7915 \\
\hline 0.4911 & 0.28 & $\mathrm{NaN}$ & 0.8 & 0.4285 \\
\hline
\end{tabular}

